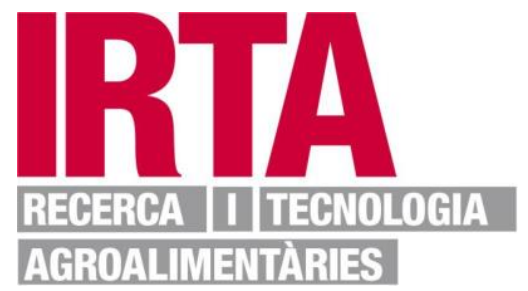

This document is a postprint version of an article published in The Journal of Nutritional Biochemistry $\subset$ Elsevier after peer review. To access the final edited and published work see https://doi.org/10.1016/j.jnutbio.2020.108393

Document downloaded from: 


\section{Molecular phenomics of a high-calorie diet-induced porcine model of}

\section{prepubertal obesity}

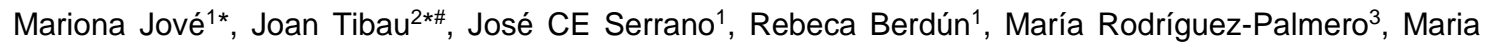
Font-i-Furnols ${ }^{2}$, Anna Cassanyé ${ }^{1}$, Reyna Rodriguez-Mortera ${ }^{1}$, Joaquim Sol ${ }^{1}$, Helene Rassendren ${ }^{1}$, Emma Fàbrega ${ }^{2}$, Anna Crescenti ${ }^{4,5}$, Anna Castell 4,5 , Mònica Sabater ${ }^{6,7,8}$ Francisco J Ortega ${ }^{6,7,8}$, Meritxell MartinGari $^{1}$, Raquel Quintanilla9 , Joaquim Puigjaner ${ }^{3}$, Jose Antonio Moreno ${ }^{3}$, Joan Prat $^{1}$, Lluis Arola ${ }^{4,10}$, Josep Manuel Fernández-Real ${ }^{6,7,8}$, Reinald Pamplona ${ }^{1}$ and Manuel Portero-Otin ${ }^{1 \#}$

${ }^{1}$ NUTREN-Nutrigenomics Fisiopatologia Metabòlica, Universitat de Lleida-IRBLleida, E25196 Lleida, Spain; ${ }^{2}$ Animal Breeding and Genetics Programme, Institute for Research and Technology in Food and Agriculture (IRTA), Finca Camps i Armet, E17121, Monells, Spain; ${ }^{3}$ Laboratorios Ordesa S.L., Barcelona Science Park , 08028 Barcelona, Spain; ${ }^{4}$ Nutrition and Health Unit, EURECAT-Technology Centre of Catalonia, Reus, Spain; ${ }^{5}$ Nutrition and Health Research Group, EURECAT-Technology Centre of Catalonia, Reus, Spain; ${ }^{6}$ CIBEROBN and Instituto de Salud Carlos III (ISCIII), Madrid, Spain; ${ }^{7}$ Institut d'Investigació Biomédica de Girona (IdIBGi), Girona, Spain; ${ }^{8}$ Department of Diabetes, Endocrinology and Nutrition (UDEN), Hospital of Girona "Dr Josep Trueta", Girona, Spain; ${ }^{9}$ Product Quality Program, Institute for Research and Technology in Food and Agriculture (IRTA), Finca Camps i Armet, E17121, Monells, Girona,Spain;10Nutrigenomics Research Group, Department of Biochemistry and Biotechnology, Universitat Rovira i Virgili, Tarragona, Spain

*Both authors should be considered first authors

E-mail:

MJ: mariona.jove@mex.udl.cat, JT: joan.tibau@irta.cat, JCES: iceserrano@mex.udl.cat, RB: rebecaberdun@gmail.com, MRP: maria.rodriguez@ordesa.es, MFF: maria.font@irta.cat, AC: annakasa4@gmail.com, RRM: In.reynarm@gmail.com, JS: jkim173@gmail.com, HR: In.rassendren@gmail.com, EF: emma.fabrega@irta.cat, AC: anna.crescenti@eurecat.org, ACastell: anna.castell@eurecat.org, MS: msabater@idibgi.org, FJO: fortega@idibgi.org, MMG: meritxell.martin@udl.cat, RQ: Raquel.quintanilla@irta.cat, JP: Joaquim.puigjaner@ordesa.es, JAMM: joseA.moreno@ordesa.es, JPrat: joan.prat@mex.udl.cat, LA: lluis.arola@urv.cat, JMFR: imfreal@idibgi.org, RP: reinald.pamplona@mex.udl.cat, MPO: manuel.portero@mex.udl.cat

\section{Running title: Characterization of porcine early obesity}

Address for correspondence: "To whom correspondence should be addressed at Animal Breeding and Genetics Programme, Institute for Research and Technology in Food and Agriculture (IRTA), Finca Camps i Armet, E17121, Monells, Spain (Joan.Tibau@irta.cat) or Fisiopatologia Metabòlica, Universitat de Lleida- 
33 IRBLleida, Edifici Biomedicina I, Avda Rovira Roure, 80 E25196 Lleida, Spain

34 (manuel.portero@mex.udl.cat)

35 Grants

36 Supported by CDTI (Centro para el Desarrollo Tecnológico e Industrial, Spain), Project reference: IPT-

37 20111008, and Generalitat de Catalunya grants 2017SGR1719 and 2017SGR696. Supported by ISCIII

38 (Instituto de Salud Carlos III, Spain), Project reference: 17-00134 cofinanced by FEDER Funds $A$ way to

39 make Europe

40 Keywords

41 lipidomics; biomarkers; insulin resistance; preclinical models

42

43 


\section{Abstract}

45 As obesity incidence is alarmingly rising among young individuals, we aimed to 46 characterize an experimental model of this situation, considering the similarity between 47 human and porcine physiology. For this reason, we fed prepubertal (63 days-old) Duroc breed females $(n=20)$ either with a standard growth diet (3800 KCal/day) or one with a high-calorie content (5200 KCal/day) during 70 days. Computerized tomography, massspectrometry based metabolomics, and lipidomics, as well as peripheral blood mononuclear cell transcriptomics, were applied to define traits linked to high-calorie intake. Samples from a human cohort confirmed potential lipidomic markers. Compared to those fed a standard growth diet, pigs fed a high-calorie diet showed an increased weight gain (13\%), much higher adiposity (53\%), hypertriacylglyceridemia and hypercholesterolemia, in parallel to insulin resistance. This diet induced marked changes in the circulating lipidome, particularly in phosphatidylethanolamine-type molecules. Also, circulating specific diacylglycerol and monoacylglycerol contents correlated with visceral fat and intrahepatic triacylglycerol concentrations. Specific lipids associated with obesity in swine (mainly belonging to glycerophospholipid, triacylglyceride, and sterol classes) were also linked with obesity-traits in the human cohort, reinforcing the usefulness of the chosen approach. Interestingly, no overt inflammation in plasma or adipose tissue was evident in this model. The presented model is useful as a preclinical surrogate of prepubertal obesity in order to ascertain the pathophysiology interactions between energy intake and obesity development.

\section{INTRODUCTION}

Childhood obesity is linked to severe metabolic derangements in adult life [1].

67 Epidemiological studies reveal alarming rates of incidence of prepubertal and pubertal obesity [2], with its worrying impact on insulin resistance, development of type 2 diabetes, increased cardiometabolic risk, decreased bone health, as well as the psychological consequences of this derangement[3]. Therefore, the burden of increased caloric unbalance could seriously affect pubertal and postpubertal health[1]. 
73 Obesity is considered a multifactorial situation [4]. Under an evolutionary point of view, adipose tissue (AT) buildup in mammals can be a physiological response over changes in calorie availability, with a signaling role $[5,6]$, which could be useful for homeostasis in an environment with energy availability oscillations. As such, increased fat content is not always linked to metabolic derangements [7]. The continuum between these two situations is still mostly unknown, leading to the uncertainty on why some individuals develop insulin resistance and enhanced cardiometabolic risk even with lack of AT buildup (metabolically unhealthy lean people) whereas other can cope with high amounts of adipose tissue (metabolically healthy obese) [8]. It is required an adequate characterization, at a molecular level, of the events between AT accumulation and its consequences, to fill this gap of knowledge.

In this line, available experimental models of obesity show some intrinsic limitations. For instance, rodents could exhibit increased obesity when placed in high-calorie intake, especially in the so-called cafeteria diet (e.g., an unbalanced diet with high fructose or high lipid content). Interestingly, due to the close physiological resemblance of porcine and human physiologies, specific cardiometabolic markers translate better in pigs than

90 in rodent models. These include low HDL-cholesterol, increased LDL-cholesterol, coronary calcification, to name a few. Thus, in both primates and swine, lipoprotein metabolism is very similar to humans $[9,10]$. Therefore, unhealthy diets in these species are able to induce changes in lipid profiles associated with cardiometabolic risk, reproducing many changes present in humans $[10,11]$. Pigs are particularly suited for evaluating their lipid metabolism, based on the knowledge of genetic traits controlling them, and the possibility to monitor the buildup of AT by using clinical imaging systems as computed tomography $(\mathrm{CT})[12,13]$. 
99 Systems biology has helped to unravel the pathogenic role of specific fat depots in both

100 humans and rodents. Among involved disciplines, proteomics, metabolomics, and

101 lipidomics are closely interlaced with the phenotype, offering a dynamic view of the 102 ongoing molecular changes. Metabolomics either focused on polar molecules or

103 lipidomics (when lipids are extracted before the analyses), have the advantage of

104 covering a high number of potential candidate biomarkers. When combined adequately

105 with confirmatory techniques, they could disclose pathogenic pathways amidst

106 generating novel hypotheses for diagnosis, prognosis, and therapeutics.

108 In this context, the present work aims to characterize molecular traits associated with the 109 obesity induced by a high-calorie diet in prepubertal female pigs. This goal is achieved

110 by combining an imaging platform based on CT evaluation of AT distribution, weight

111 evolution, food intake with changes in circulating lipidome and metabolome, with a view

112 of specific transcripts in the subcutaneous AT and in the transcriptome of the peripheral

113 blood mononuclear cells (PBMCs). These later were chosen as they may share, at least

114 partially, share the expression profile of different sets of genes with other tissues,

115 including those that reflect metabolic responses[14-16]. Furthermore, the lipidomic

116 changes were validated in a cohort of human subjects to confirm the robustness of these

117 observations. Finally, these changes were compared with clinically validated biomarkers

118 of obesity-related dyslipidemia with some of the suggested pathogenic pathways 119 confirmed in adipose and liver tissues.

2. METHODS

122 All the experimental procedures with pigs, including management, traits recording,

123 monitoring, and blood sampling, were approved by the Ethical Committee of the Institut

124 de Recerca i Tecnologia Agroalimentàries (IRTA, Girona, Spain). In the case of human 125 samples, these were obtained from the FLORINASH study cohort [17], and its 126 institutional review board approved the study protocol, and all subjects provided informed 
127 written consent. Briefly, we recruited 44 consecutive subjects. Inclusion criteria were age

$128 \quad 30-65$ years and acceptance to study procedures. Exclusion criteria were a systemic

129 disease, infection in the previous month, chronic severe illness, ethanol intake $>20 \mathrm{~g} /$ day,

130 or use of medications that might interfere with insulin action. For comparison with porcine

131 model-derived biomarkers, we recorded body to mass index (BMI) and homeostatic

132 model assessment for insulin resistance (HOMA-IR). See supplemental Table 1 for

133 clinical characteristics (supplemental data). Sources for chemicals are also presented in

134 the extended Methods section (supplemental data).

\section{$135 \quad 2.1$ Animals and treatments}

136 A total of 21 female piglets from a high intramuscular fat Duroc pig line were used in the

137 present study. Animals were born in 10 different litters (i.e., ten pairs of littermates) and 138 were distributed randomly to one of the two diets (conventional diet for growth and 139 western-type, see below). After weaning, piglets were transferred to the IRTA pig 140 experimental test station and subjected to the same management procedures. From 141 weeks 9 to 11, animals were adapted to a transition diet (Supplemental Tables 2 and 3).

142 Subsequently, each of the sibling pairs was divided into the two dietary treatments:

143 animals fed a conventional growth diet and animals fed a western-type diet with a high-

144 calorie, high-sucrose and low fiber content (detailed composition and nutritional 145 properties shown in supplemental Tables 4 and 5, ). The experiment lasted until pigs 146 reached 19 weeks of age when animals were sacrificed after an overnight fast, at IRTA 147 experimental slaughterhouse compliance with all welfare regulations. Pigs were weighed 148 individually at the beginning and every two weeks during the whole experiment, plus the 149 day before slaughter. Daily feed intake (FI; $\mathrm{kg} /$ day) was recorded individually through 150 theautomatic electronic feeding system HOKOFARM-IVO-G ® (Marknesse, The

151 Netherlands): all animals are identified electronically and located in contiguous pens in 152 the same barn (with $10-11$ animal groups of littermates). Each pen is provided with an 153 automatic feeding recording system (system HOKOFARM-IVO-G $®$ ), allowing full control 
154 of individual voluntary ad libitum feed intake. Each particular meal intake ( 10 to 20 per

155 day) is recorded, and the full dailyfeed ingested amount is computed for each animal (as

156 well as the number and duration of meals). Blood samples for biochemical and

157 transcriptome analyses were taken from fasted animals immediately before sacrifice.

158 After slaughtering, pelvic-renal fat (flare fat) was removed and weighted.

\subsection{Computed tomography scanning and image analysis}

160 All pigs were CT-scanned one week before sacrifice employing the General Electric HiSpeed ZX/I (Fairfield, CN, USA) equipment located at IRTA in Monells (Girona,Spain) as explained in [12] and images were analyzed using software VisualPork $[18,19]$

163 developed by the University of Girona and IRTA. See Figure 1A and 1B as an example 164 of employed images. See the extended Methods section (supplemental data) for further 165 description.

\subsection{Biochemical analyses and western blot}

168 Serum lipids and other conventional biochemical variables were measured in fasted animals in blood obtained immediately before sacrifice by using commercial kits,

170 including triacylglycerides, total cholesterol, LDL- cholesterol, HDL cholesterol, and

171 glucose, as described previously [20]. Plasma interleukin concentrations were measured

172 using Millipore multiplex kits for porcine samples (Millipore, Barcelona, Spain) according

173 to manufacturer instructions. Plasma insulin content was measured using a commercial

174 ELISA kit (Abcam, Cambridge, UK). Total lipids were extracted from the liver (80 mg)

175 and dried feces $(100 \mathrm{mg})$ of the pigs and were quantified using the methods previously described [21]. In the liver and feces samples, triglycerides and cholesterol were quantified by colorimetric kits (QCA, Barcelona, Spain). Western blot analyses of tissues

178 were performed as described[20] and explained in the extended Methods section 
179 (supplemental data), using as primary and secondary antibodies those listed in

180 supplemental Table 6 (supplemental data).

$181 \quad 2.4$ Untargeted lipidomic analyses of plasma

182 Plasma lipidomic analysis was based on a previously validated method [22]. Internal 183 standards (listed in supplemental Table 7), lipid extraction, and lipidomic techniques are 184 explained in the extended Methods section (supplemental data). Lipid extracts were 185 subjected to liquid chromatography coupled to mass spectrometry (LC-MS) using an 186 Agilent UPLC 1290 coupled to the Q-TOF MS/MS 6520 (Agilent Technologies, 187 Barcelona, Spain) based on a previously published method [23].

\subsection{Untargeted metabolomics analyses of serum}

189 The metabolomic analyses of serum extracts were performed by LC-MS and further 190 explained in the extended Methods section (supplemental data).

1912.6 Peripheral blood monocyte cells (PBMC) transcriptomics and AT leptin 192 expression

193 PBMCs transcriptomics was done of ( $n=7$ from each group) at the Centre for Omic 194 Sciences (Reus, Spain) by using the Porcine (V2) Gene Expression Microarray, 4x44K 195 (Agilent Technologies) following manufacturer's instructions. AT mRNA expression in 196 the subcutaneous depot was quantified as previously described [24] by using real-time

197 PCR in a LightCycler 480 Real-Time PCR System (Roche Diagnostics) using TaqMan®

198 technology suitable for relative gene expression quantification. Both methods are further 199 developed in the extended Methods section (supplemental data).

\section{$200 \quad 2.7$ Data processing, Network, and Statistic Analyses}

201 In the case of lipidomics and metabolomics, the MassHunter Data Analysis Software 202 (Agilent Technologies) was used to collect the results and the MassHunter Qualitative 203 Analysis Software (Agilent Technologies) to obtain the molecular features of the 204 samples, representing different, co-migrating ionic species of a given molecular entity 205 (i.e., ion adducts) using the Molecular Feature Extractor algorithm (Agilent Technologies) 
206 [25]. This algorithm uses the accuracy of the mass measurements to group related ions

207 (based on the charge-state envelope, isotopic distribution, and the presence of different

208 adducts and dimers/trimers) assigning multiple species (ions) to a single compound 209 referred to as a feature. Finally, the MassHunter Mass Profiler Professional Software

210 (Agilent Technologies) was used to perform a non-targeted analysis over the extracted

211 features. Only shared features (found in at least $75 \%$ of the samples of the same

212 condition) were taken into account to correct for individual bias. In the case of non-

213 targeted lipidomics, proposed annotation by lipid class is based on exact mass, isotopic

214 distribution, and retention time of lipid family standards, so fatty acid distribution offered

215 is one of the potential combinations leading to the same number of carbons and 216 unsaturations.

217 In the case of non-targeted metabolomics, the identification of detected compounds with

218 significant differences was performed based on the exact mass, the adduct formation,

219 and the isotopes relative abundance and spacing using the Metlin/PCDL database and

220 Molecular Formula Generation software (Agilent Technologies).

221 In case of lipidomics, after log transformation and auto-scaling of variables, the masses representing significant differences by Student's t-test $(p<0.05$ with false discovery rate correction) were searched against the LIPID MAPS database (The LIPID MAPS

224 Lipidomics Gateway, http://www.lipidmaps.org/, February 2018) (exact mass ppm < 20).

226 All other analyses were performed either with the SAS software ver. 9.4 (SAS Institute 227 Inc., Cary, NC, USA), with the Prism ver 7 (GraphPad Software, La Jolla, CA, USA) or with the SPSS software ver 24 (IBM Corp, Armonk, NY, USA). Non-linear fit (exponential growth equations; $\left.\mathrm{Y}=\mathrm{Y} 0^{*} \exp \left(\mathrm{k}^{*} \mathrm{X}\right)\right)$ lines in the graphs correlating two variables were

230 produced with the Prism software. Multivariate statistics (Hierarchical Clustering, PCA, 231 and PLS-DA analyses) were done using the Metaboanalyst platform software [26]. Gene 232 enrichment and induced network module analyses were performed in the 
233 ConsensusPathDB platform [27]using as input genesagtpbp1, fcrl4,herc4,egr2,

234 loc780435, loc100622399, loc100621244, krt18, ly49, pth, kctd14, ppp1r36, and trim46.

235 The same list was also explored with the Genemania platform[28], using Homo sapiens

236 (human) as the reference and searching for its potential relationship with genes in lipid

237 metabolism pemt and srbep1.

238

\section{RESULTS}

240 As expected, animals under the western-type regime increased weight in a significant

241 amount (Table 1). However, despite these animals ingested near 37\% more calories per

242 day, the difference in weight did not reach these changes, only achieving $13 \%$ more

243 weight. This ponderal change was also associated with an increased fecal fat content (in

244 cholesterol and triacylglyceride content). Nonetheless and despite these data pointing

245 for a trend for fecal lipid loss, adiposity, measured by CT imaging, increased sharply,

246 reaching ca two fold increases in AT derived parameters, such as relative fat volume and

247 pelvic-renal fat content. Concerning biochemical consequences associated with obesity,

248 animals under the high-calorie regime increased plasma cholesterol significantly, its

249 subfractions, and triacylglyceridemia in parallel to insulin resistance (HOMA-IR, ca 43\%,

$250 \mathrm{p}<0.01$ ), suggesting an early impairment in glucose homeostasis (Table 1).

252 To enhance the knowledge of the molecular basis of this phenotype, we performed a non-targeted lipidomic analysis in plasma samples. This approach shows that prepubertal obesity changed the amounts of 187 molecules ( $p$ values ranging between 1.49E-20 and 0.05; Supplemental dataset 1), with very significant changes in 23 compounds (FDR <0.1). These comprised 4 phosphatidylethanolamines and a cerebroside, among the top markers. Hierarchical clustering resulted in a clear separation between the two lipidomes using the 25 most statistically significant molecules (Figure 1C) with a PLS-DA model (Figure 1D) with high accuracy $(71 \%$, with 
$260 \mathrm{R} 2=0.92$ in a one model component, Supplemental Figure 1). Variable importance in

261 projection scores reinforced the importance of several phosphatidylethanolamine and

262 phosphatidylserine molecules in the first component, with a cerebroside, a 263 monoacylglycerol and a sterol related molecule among these discriminating features

264 (Supplemental Figure 1).

265

266 We studied if specific circulating lipids correlated with individual parameters affecting

267 final weight since the high-calorie intake impacted differently on each individual (e.g.,

268 variances higher than $10 \%$ in the final weight, Table 1). Concerning calorie consumption,

269218 molecules correlated significantly with calorie intake ( $p$ values ranging between

$2707.61 \mathrm{E}-7$ and 0.05 , Spearman rank correlation between absolute values between 0.85

271 and 0.42, Supplemental Dataset 2). Among these, three phosphatidylethanolamines

272 were among the top targets correlating with calorie intake (Figure 1D). Interestingly,

273 several molecules also correlated with AT content (226 molecules, with $p$ values

274 between $6.8 \mathrm{E}-6$ and 0.05 , Spearman rank absolute correlation values between 0.81 and

275 0.43, Supplemental Dataset 3). Besides phosphatidylethanolamines, other lipids showed

276 a high correlation, such as a monoglyceride and a cerebroside (Figure 1E). The targets

277 correlating with pelvic-renal AT depot abundance were different from those of total fat,

278 suggesting the fact that non-subcutaneous fats constitute depots with unique

279 pathophysiological traits. Thus, 169 different molecules correlated with pelvic-renal

280 depot abundance ( $p$ values between $5.9 E-5$ and 0.05 , Spearman rank absolute

281 correlation values between 0.76 and 0.43 , Supplemental Dataset 4). Though

282 phosphatidylethanolamines were correlated as well, top molecules belonged to

283 cholesteryl esters and to phosphatidylserine (Figure 1F).

285 Interestingly and independently from lipidomic changes in plasma, a partial least square

286 discriminant analyses (PLS-DA) model encompassing AT depots and lipidemia variables

287 ((plasma, liver, and LDL cholesterol) exhibit a high accuracy (Supplemental Figure 2A). 
288 Clustering analyses showed that not all the adipose tissue depots clusterized together,

289 with pelvic-renal fat being associated with lipidemia variables, (Supplemental Figure 2B).

290 In contrast, total fat and subcutaneous fat depot were more closely associated with

291 calorie intake and final weight. Variable importance in projection (Supplemental Figure

292 2C) showed that the most influential variables were calorie intake, total fat, and

293 subcutaneous fat percentages. Permutation tests demonstrated the robustness of this

294 model (Supplemental Figure 2D).

296 As LDL cholesterol was also affected by western-typeregime, we evaluated potential 297 lipidomic markers linked to this biochemical trait. Interestingly, these differed from those correlated with other biochemical characteristics. Thus, 165 molecules correlated with

299 LDL cholesterol concentrations ( $p$ values between 2.5 E-5 and 0.05, Spearman rank

300 correlation absolute values between 0.71 and 0.43 , Supplemental DataSet 5). These

301 molecules comprised a specific phosphatidylethanolamine (different from the one

302 correlated with fat contents) and several unknown lipids (Supplemental Figure 3).

304 As increased fasting glycemia and HOMA-IR (despite no overt hyperinsulinemia) were evidenced in this model of prepubertal obesity, we searched for lipidomic markers of

306 insulin resistance. Insulin concentrations were correlated with 90 different lipids ( $p$ values

307 between 0.0003 and 0.05 , Spearman rank absolute correlation values between 0.68 and

308 0.43, Supplemental Dataset 6). Specific molecules comprised a phosphatidic acid and a

309 lactosyl-ceramide (Figure 2A). Similarly, a total of 77 molecules correlated with HOMA-

310 IR ( $p$ values between 0.0002 and 0.05 , Spearman rank absolute correlation values

311 between 0.75 and 0.46 , Supplemental Dataset 7). These included specific

312 triacylglycerides and sphingolipids (Figure 2A). Insulin acts on adipogenesis via specific

313 effectors such as SREBP1 [29]. Although we did not observe overt hyperinsulinism but

314 insulin resistance, we evaluated insulin-dependent signals in adipose tissues of this

315 model. The results demonstrate anatomic differences in the impact of a high-calorie diet 
316 in insulin signaling modules of adipose tissue. Both adipose tissues from pelvic-renal

317 and omental locations showed a significantly increased amount of SREBP1c, while

318 subcutaneous and omental ATs locations showed decreased Glut4 levels, consistent

319 with insulin resistance (Figure 2B). Of note, AT in the pelvic-renal location did not show

320 detectable levels of Glut4 (data not shown), but Glut2. We also evaluated the expression

321 of specific mRNs in the subcutaneous depot (Supplemental Table 8). We studied which

322 of the circulating lipids correlated with leptin expression since leptin levels mirror

323 adipogenesis in subcutaneous depot (significantly increased by evaluation of its mRNA

324 content in $186 \%, \mathrm{p}<0.00001)$. The results demonstrate significant associations of leptin

325 mRNA levels in subcutaneous AT and 138 lipids in plasma ( $p$ values between 4.92E-05

326 and 0.049 , Spearman rank correlation absolute values between 0.76 and 0.43 ,

327 Supplemental Dataset 8). These include specific phosphatidylethanolamine and

328 triacylglycerides (Figure 2C). The specificity of the lipidomic changes associated with

329 insulin signaling is evidenced by the lack of common lipidomic correlations with leptin

330 mRNA and very low common correlations with fat content (Figure 2D).

331 As the liver is also a target of obesity, we evaluated the potential occurrence of hepatic

332 steatosis in this model. The results show that the western-type diet in this model did not

333 change neither triacylglycerides nor cholesterol content (Supplemental Figure 4).

334 Despite no significant changes were measured in phosphorylated-IRS1 (Ser 307, Ser

335 1101) or in phosphorylated c-Jun (Ser 73) (supplemental Figure 4) decreased Glut2

336 content potentially marked incipient insulin resistance, (Figure 3A), with some circulating

337 lipids correlating significantly with changes in hepatic fat content. Thus, 123 molecules

338 correlated with hepatic triacylglyceride content ( $p$ values between $1 E-4$ and 0.05 ,

339 Spearman rank absolute correlation values between 0.74 and 0.43 , Supplemental

340 Dataset 9). These comprised a diacylglycerol and a phosphatidic acid (Figure 3B).

341 Plasma species correlating with liver triacylglyceride content are quite specific (Figure

$3423 \mathrm{C})$. Noteworthy, changes in glucose transporters did not affect, at this stage, skeletal

343 muscle (Supplemental Figure 5). 
345 In line with the moderate impact of high calorie in this model, plasma values of measured

346 interleukins did not demonstrate overt inflammation (Supplemental Table 9). However,

347 statistically significant correlations were present between circulating lipids and selected

348 interleukins, including IL-1a, IL-1b, and IL-18 (Supplemental figure 6, supplemental

349 Dataset 10 for correlated lipids). These lipids comprised specific

350 phosphatidylethanolamines, triacylglycerides, and tocopherol derivates.

352 To evaluate whether this model could be of usefulness in human obesity, we compared

353 the results with those obtained from plasma lipidomic analyses of a human cohort. As

354 Figure 4 shows, there is a consistent overlap between the behavior of selected

355 biomarkers of obesity and its metabolic consequences between these two species. Thus,

356 selected phospholipid molecules (phosphatidylserine $(21: 0,22: 0)$, sterols (5-cholestan-

357 3-one), and unidentified lipids correlated significantly both with BMI in humans and with

358 fat abundance in pigs. Interestingly, other lipids also correlated with HOMA-IR in both

359 species, underlying the usefulness of the chosen model. The same behavior was

360 observed for correlations between cerebroside $\mathrm{D}, \mathrm{N}-(11 \mathrm{Z}, 14 \mathrm{Z}$-eicosadienoyl)-

361 ethanolamine, TG $(16: 0 / 16: 1(9 Z) / 16: 1(9 Z))$ and $B M I\left(R^{2}=0.31, p<0.0001 ; R^{2}=0.09\right.$,

$362 \mathrm{p}<0.03 ; \mathrm{R}^{2}=0.12, \mathrm{p}<0.02$ after Pearson correlation analyses), as presented in

363 Supplemental Figure 7.

364

365 To further characterize the impact of western-type diet in the porcine model, we also

366 analyzed changes in the aqueous soluble phase of serum and explored changes in the

367 PBMC transcriptome profile. Globally speaking, the number of differential features was

368 much lower comparing the aqueous extracts with those of organic solvents

369 (Supplemental Table 8). When aggregating these changes of metabolites into pathways,

370 these defined a network that comprised variations in metabolites belonging to branched-

371 chain amino acids (BCAA), central carbon metabolism, and pantothenate and coenzyme 
372 A synthesis (Figure 5). Changes in PBMC transcriptome (Supplemental Dataset 11), 373 when mapped, revealed two main networks affected by high-calorie intake

374 (Supplemental Figure 8). One was related to EGR2, and the other was mainly paired 375 with KRT18 and RAAR genes. The functional annotation of these genes clustered in 4 376 different enriched neighborhood-based tests $(\mathrm{p}<0.001)$, which included SUV420H2, 377 DTX4, DTX1, and USP49 (Supplemental Figure 9). We also evaluated the potential 378 network proximity between genes in PBMC transcriptome and key genes in metabolism, 379 SREBP1, and PEMT. These two later belonged to the network of expression of KRT18 380 and EGR2 (Supplemental figure 10).

\section{DISCUSSION}

383 The present work reinforces the usefulness of high-calorie regimes in porcine models to 384 evaluate obesity pathophysiology [30,31]. Interestingly, we found that the body weight 385 gain induced by high calorie, although significant, was not proportional to calorie intake. 386 This fits with the notion that weight gain and calorie intake are not linear[32]. In rats, 387 physical activity, among other factors, could divert dietary fats towards oxidation and less to the storage, in a manner associated with de novo lipogenesis in adipose tissue, as well as increases in skeletal muscle uptake and oxidation of lipids [33]. This metabolic switch seems not uncommon in swine [34]. Of note, fat content increased in the highcalorie group. In any case, our model can be viewed as an early obesity model, with some traits of metabolic syndrome. This resemblance with human pathology has been already described for domestic pigs under similar, high-fat regimes, where short term interventions led to weight gain and dyslipidemia, but not tovert insulin resistance or metabolic syndrome[35]. Several factors may explain the relatively small metabolic impact in the current porcine model in comparison to other reported porcine models,

397 including the age of the individuals, the lack of a large amount of refined sugars (fructose 398 or sucrose), in comparison to other diets inducing obesity in porcine models, the specific 399 breed or the length of treatment. 
401 In order to characterize the molecular traits explaining these obesity-related changes, 402 we performed non-targeted lipidomic analyses of plasma samples. For the sake of 403 brevity, we will limit mainly the discussion of those results obtained after FDR correction.

404 The results suggest the role of specific phosphatidylethanolamines as potential obesity 405 circulating biomarkers at a molecular level. One of the most differential identifiable 406 markers is a dimethylphosphatidylethanolamine. These products are intermediates in 407 the liver synthesis of phosphatidylcholine de novo biosynthesis through methylation of 408 phosphatidylethanolamine, especially relevant when choline is deficient in diet [36]. This conversion is catalyzed by the enzyme phosphatidylethanolamine $N$-methyltransferase

410 (PEMT), whose activity is regulated by adenosylmethionine and

411 phosphatidylethanolamine levels. Since this activity is essential for VLDL secretion, we

412 hypothesize that increased levels of the dimethylphosphatidihylethanolamine detected 413 could be a proxy for increased PEMT activity in the liver and VLDL secretion, required 414 for exporting hepatic lipids towards their adipose depot. PEMT loss renders protection 415 over diet-induced obesity[37], suggesting that in our model of obesity, this activity is 416 increased. Noteworthy, PEMT expression and colocalization are related with EGR2 and $417 K R T 18$, as pathway analyses show. Other lipids significantly related to energy intake, 418 were specific phosphatidylethanolamine (PE) with long fatty acids. These findings could 419 go in line with data from lipidomic analyses of plasma from overweight and obese healthy 420 individuals [38]. In these analyses, other PE species appeared to be relevant 421 components of a plasma lipidomic signature of obesity. Interestingly, in other cohorts of 422 patients with prediabetes and diabetes, increased PE levels were noted as well [39]. In 423 this context, these were interpreted as a source for inflammatory eicosanoids, which may 424 be as well our case, as one of the potential fatty acids present in these PE is arachidonic 425 acid. Other works revealed an association of a specific PE, containing 40:6 fatty acids, 426 with insulin resistance in human individuals[40]. 
428 Reinforcing the validity of porcine lipid metabolism in the context of human obesity,

429 increases in specific PE-related species[41], such as plasmalogens, were observed in 430 obese subjects. Plasmalogens are part of an antioxidant response towards increased 431 oxidative stress with high-calorie intake. Nonetheless, several phospholipids 432 (phosphatidylserine and sterol molecules) correlated with BMI and AT abundance in 433 both species using the same methodological approach.

435 Other lipids were significantly associatedwith adiposity, measured by CT imaging. Thus, 436 increases in a long chain monoacylglycerol were detected. In mice, the deletion of one 437 of the enzymes linked to monoacylglycerol degradation, monoacylglycerol lipase, leads 438 to increased concentrations of monoacylglycerol and resistance to obesity, as well as 439 delayed lipid absorption [42]. The increased levels detected here may be viewed as a 440 potential homeostatic downregulation of monoacylglycerol lipase activity. Another lipid 441 showing a similar behavior with adiposity, cerebroside $D$, has been reported to be a 442 potent immunomodulatory agent in mice models of experimental colitis[43]. Furthermore, 443 glucocerebrosides afford protection over the metabolic syndrome induced by leptin loss 444 in mice [44]. It is known that structurally related compounds, such as glucosylated 445 ceramides and ceramides, play critical roles in insulin resistance, which has an 446 inflammatory background [45], therefore increased cerebroside D might be viewed as a 447 compensatory response to avoid the high-energy induced inflammatory burden. Of note, 448 cerebroside $\mathrm{D}$ was also found in the humane cohort used for the translational applicability 449 of the model, thereby reinforcing its potential importance in obesity pathophysiology.

451 In line with the pathophysiological relevance of the location of adipose tissue depots 452 [24], the lipids that correlated with total adiposity were different from those correlating 453 with the amount of pelvic-renal fat depot, a typical visceral location. Cholesteryl ester $45418: 3$, associated with the amount of this AT, has been previously shown to be decreased 455 in a murine model of obesity [46]. However, and reinforcing the similarity of porcine 
456 models with humans, a sizeable lipidomic survey revealed a significant association of 457 cholesteryl esters with body mass index in humans[47]. The other lipid, a 458 phosphatidylserine, has not been previously related to obesity, but instead to 459 preeclampsia in humans[48]. Glycerophosphoserines play vital structural roles in cell 460 membranes [49], where they may regulate oxidative stress responses and apoptosis, 461 but so far, it was not associated with high energy intake. Of note, a glycerophosphoserine 462 was found among the biomarkers shared between the porcine study and the humane 463 cohort. These lipids have been linked recently to changes in postprandial lipidome after 464 physical activity in humans with type 2 diabetes [50].

466 Finally, phosphatidic acid and diacylglycerol were associated with triacylglyceride 467 content in the liver. Interestingly, diacylglycerol species were as well noted as relevant 468 biomarkers by overfeeding experiments in humans[41], and phosphatidic acid increases 469 considerably during diet-induced nonalcoholic fatty liver disease [51]. Further, these data 470 reinforce the usefulness of plasma lipidomic signature for minimally invasive monitoring 471 of liver status [52].

472

473 Even though we detect no changes in some circulating surrogates of insulin resistance 474 (such as fructosamine), we were able to detect molecular markers of adipose tissue and 475 liver insulin resistance. Sphingolipids were found among the measured markers 476 correlating with HOMA-IR and insulin. This relationship agrees with the well-described 477 role of ceramide signaling in human obesity-related insulin resistance (reviewed in[53]). 478 Globally, this association would reinforce the validity of this model in evaluating the 479 pathophysiology of obesity-related metabolic rearrangements. In line with this, and 480 similar to humans, fat depots showed a location-specific response to high-calorie diet in 481 the expression of glucose transporters and adipogenic proteins (as SREBP-1c). Further, 482 AT leptin expression was increased, in line with adipose tissue expansion. 
484 The changes in circulating metabolome and transcriptome also support the usefulness

485 of the model: changes in BCAA metabolism have been described in several clinical 486 studies, demonstrating that its alteration could predict diabetes development[54]. These 487 variations can be associated with ongoing inflammation and with mitochondrial 488 alterations, though in our model, no evidence for inflammation was found. Interestingly, 489 we also found pieces of evidence for altered pantothenic-related metabolic pathways in 490 our model of obesity. This concept fits with recently described findings in Yucatan mini491 pigs fed with a high-fat,high-sucrose diet for two months, also reporting amino acid 492 metabolism changes, reduced capacities of BCAA transamination, and alteration in 493 pantothenic metabolism [55]. In line with these results, transcriptomic changes in PBMC 494 agree with previous data in humans. Thus, previous data in adipocytes reveal that EGR 495 function, related to one of the pathways found in the transcriptome, is required to 496 enhance triacylglyceride depot [56]. In mice, EGR represses FOXC2 expression and is 497 required for the development of obesity under high-calorie intake. Of note, it is known 498 that insulin inhibits EGR expression in peripheral cells [57]. Therefore, one could see 499 EGR changes in PBMC as early markers for insulin dysfunction. Similarly, KRT18 has 500 been invoked as a biomarker for obesity-related hepatic dysfunction in obese 501 adolescents [58], so our finding of its change in this model would support its use as a

502 preclinical model of human pubertal obesity. Functional neighborhood analyses revealed 503 ubiquitin ligases DTX4 and DTX1 and the methyltransferase SUV420h2 as potential 504 nodes of interest, as previously reported data on adipocyte differentiation and diet505 induced obesity reveals $[59,60]$. These results reinforce the usefulness of PBMC 506 transcriptome analyses in this model.

508 We assume, as limitations of the present work, that only using a single-gender could 509 hinder the application of those results to human pathophysiology, especially those 510 related to the influence of sex hormones in lipid metabolism. However, the fact that many 511 markers were replicated in a completely independent fashion in a human cohort (with 
512 both genders and older age) with obesity supports the usefulness of the chosen

513 approach. Further, we acknowledge that a more thorough examination of this cohort

514 (e.g., at older ages) would be advantageous to define the unique features of

515 prepubescent obesity in this context. Based on the comparative analyses with the human

516 cohort, we propose that those markers in common with consolidated obesity could show

517 some promise in the pathophysiological research of increased AT depots. In contrast,

518 those not in common with humans could be either derived from specific porcine

519 metabolism, from younger age of examined specimens, or related to resilience towards

520 pathological effects of AT buildup. Although the number of calories consumed is much

521 higher in comparison with humans (both in the conventional and in the western-type

522 diet), the experimental group exhibited a marked increase in daily intake of calories.

523 Further, on the qualitative side, experimental diets modeling western-type diets include

524 those such as the "cafeteria diet" with high-calorie content, high sucrose, and fat

525 contents, with lower protein amounts $[61,62])$. These also include high fat with high

526 cholesterol content[63]. Other authors also include a low fiber component[64]. Despite

527 this diversity, our model shows most of these traits, reproducing the western-type dietary

528 patterns. Further, lipidomic identification proposed aims at level 2, according to the

529 Metabolomics Standards Initiative [65], so they are putatively annotated compounds

530 (e.g., without chemical reference standards, based upon physicochemical properties and

531 spectral similarity with public/commercial spectral libraries). Therefore, some of these

532 reported metabolites might vary in their final identification., Noteworthy, our model of

533 prepubescent obesity may be useful for the evaluation of the biological basis of

534 metabolically healthy obesity. A high proportion of obese children are metabolically fit

535 [66]in a close relationship with a higher propensity of subcutaneous fat accumulation.

536 Our model shows both a high subcutaneous fat accumulation concurrently with a non-

537 marked inflammatory response. Further, some of the circulating lipids correlate with

538 physiological responses to AT expansion (e.g., leptin expression), while some others

539 correlate with pathological traits (inflammatory markers or the HOMA-IR). This fact 
540 reveals the mixed nature of lipidomic responses to western-type diet in this model, but

541 we think that the reported data could be of interest in order to get the full profit of this

542 model as a bona fide preclinical surrogate of pubertal obesity.

544 COMPETING INTERESTS

$545 \quad$ No competing interests declared.

\section{FUNDING}

547 Supported by CDTI (Centro para el Desarrollo Tecnológico e Industrial, Spain), Project

548 reference: IPT-20111008, and Generalitat de Catalunya grants 2017SGR1719 and 549 2017SGR696. Supported by ISCIII (Instituto de Salud Carlos III, Spain), Project 550 reference: $17-00134$ cofinanced by FEDER Funds $A$ way to make Europe

\section{AUTHORS CONTRIBUTIONS}

553 Conceptualization:J.T.,M.R.P,J.Pu.,J.Pr,M.P.O.

554 Methodology:M.J.,J.T.,M.F.F.,J.Pu,M.P.O.

555 Software:M.F.F.,J.T.,J.S., E.F.

556 Validation:R.B.,L.A., M.M.G.,J.M.F.R.

557 Formal analysis:M.J., J.C.E.S.,J.S.,A.Cr., A. Cast,M.S.,F.J.O.,L.A., J.M.F.R.

558 Investigation:M.J., J.C.E.S.,A.C.,H.R., A. Cr.,R.R.M., A.Cast.,M.S.,R.Q.

559 Resources:M.R.P., J.T.,R.Q.,J.M.F.R.

560 Data curation:A.C., J.S., H.R.,E.F.

561 Writing - original draft preparation:M.P.O.,J.A.M.,J.T.

562 Writing - review, and editing:R.Q.,J.A.M., J. Pr, J.M.F.R., R.P., M.P.O

563 Visualization:R.P,M.F.F., E.F.

564 Project administration:J.T.,M.R.P

565 Funding acquisition:J.T.,M.R.P.,J.M.F.R., M.P.O

\section{SUPPLEMENTARY DATA}

567 All supplementary figures and tables are present as supplementary information 
available at Journal's website. Supplemental Datasets are freely available under

a CC BY 4.0 license at Figshare.com website at

570 https://figshare.com/s/bf91f3332d1f6d9d4f26

\section{REFERENCES}

573

574

575

576

577

578

579

580

581

582

583

584

585

586

587

588

589

590

591

592

593

594

595

596

597

598

599

600

601

602

603

604

605

606

607

608

609

610

611

612

613

614

615

616

[1] Liang Y, Hou D, Zhao X, Wang L, Hu Y, Liu J, et al. Childhood obesity affects adult metabolic syndrome and diabetes. Endocrine 2015;50:87-92. doi:10.1007/s12020-015-0560-7.

[2] Cunningham SA, Kramer MR, Narayan KMV. Incidence of childhood obesity in the United States. N Engl J Med 2014;370:403-411.

doi:10.1056/NEJMoa1309753.

[3] Deckelbaum RJ, Williams CL. Childhood obesity: the health issue. Obes Res 2001;9 Suppl 4:239S-243S. doi:10.1038/oby.2001.125.

[4] Procter KL. The aetiology of childhood obesity: a review. Nutr Res Rev 2007;20:29-45. doi:10.1017/S0954422407746991.

[5] Trayhurn P, Beattie JH. Physiological role of adipose tissue: white adipose tissue as an endocrine and secretory organ. Proc Nutr Soc 2001;60:329-339. doi:10.1079/PNS200194.

[6] Trayhurn P, Bing C. Appetite and energy balance signals from adipocytes. Philos Trans R Soc Lond B, Biol Sci 2006;361:1237-1249. doi:10.1098/rstb.2006.1859.

[7] Bervoets L, Massa G. Classification and clinical characterization of metabolically "healthy" obese children and adolescents. J Pediatr Endocrinol Metab 2016;29:553-560. doi:10.1515/jpem-2015-0395.

[8] Phillips CM. Metabolically Healthy Obesity: Personalised and Public Health Implications. Trends Endocrinol Metab 2016;27:189-191. doi:10.1016/j.tem.2016.02.001.

[9] Litten-Brown JC, Corson AM, Clarke L. Porcine models for the metabolic syndrome, digestive and bone disorders: a general overview. Animal 2010;4:899-920. doi:10.1017/S1751731110000200.

[10] Miller ER, Ullrey DE. The pig as a model for human nutrition. Annu Rev Nutr 1987;7:361-382. doi:10.1146/annurev.nu.07.070187.002045.

[11] Hamamdzic D, Wilensky RL. Porcine models of accelerated coronary atherosclerosis: role of diabetes mellitus and hypercholesterolemia. J Diabetes Res 2013;2013:761415. doi:10.1155/2013/761415.

[12] Carabús A, Gispert M, Brun A, Rodríguez P, Font-i-Furnols M. In vivo computed tomography evaluation of the composition of the carcass and main cuts of growing pigs of three commercial crossbreeds. Livest Sci 2014;170:181-192. doi:10.1016/j.livsci.2014.10.005.

[13] Lucas D, Brun A, Gispert M, Carabús A, Soler J, Tibau J, et al. Relationship between pig carcass characteristics measured in live pigs or carcasses with Piglog, Fat-o-Meat'er and computed tomography. Livest Sci 2017;197:88-95. doi:10.1016/j.livsci.2017.01.010.

[14] Reynés B, van Schothorst EM, Keijer J, Palou A, Oliver P. Effects of cold exposure revealed by global transcriptomic analysis in ferret peripheral blood mononuclear cells. Sci Rep 2019;9:19985. doi:10.1038/s41598-019-56354-6.

[15] Zeng Y, David J, Rémond D, Dardevet D, Savary-Auzeloux I, Polakof S. Peripheral Blood Mononuclear Cell Metabolism Acutely Adapted to Postprandial Transition and Mainly Reflected Metabolic Adipose Tissue Adaptations to a HighFat Diet in Minipigs. Nutrients 2018;10. doi:10.3390/nu10111816. 
[16] Cifre M, Palou A, Oliver P. Cognitive impairment in metabolically-obese, normalweight rats: identification of early biomarkers in peripheral blood mononuclear

[17] Lelouvier B, Servant F, Païssé S, Brunet A-C, Benyahya S, Serino M, et al. Changes in blood microbiota profiles associated with liver fibrosis in obese patients: A pilot analysis. Hepatology 2016;64:2015-2027. doi:10.1002/hep.28829.

[18] Boada I, Spinola J, Rodriguez J, Martínez R. VisualPork towards the simulation of a virtual butcher. II Workshop on the Use Of ... 2009.

[19] Bardera A, Martínez R, Boada I, Font-i-Furnols M. VisualPork towards the simulation of a virtual butcher. FAIM I Conference of COST ... 2012.

[20] Serrano JCE, Gonzalo-Benito H, Jové M, Fourcade S, Cassanyé A, Boada J, et al. Dietary intake of green tea polyphenols regulates insulin sensitivity with an increase in AMP-activated protein kinase $\alpha$ content and changes in mitochondrial respiratory complexes. Mol Nutr Food Res 2013;57:459-470. doi:10.1002/mnfr.201200513.

[21] Hara A, Radin NS. Lipid extraction of tissues with a low-toxicity solvent. Anal Biochem 1978;90:420-426. doi:10.1016/0003-2697(78)90046-5.

[22] Pizarro C, Arenzana-Rámila I, Pérez-del-Notario N, Pérez-Matute P, GonzálezSáiz J-M. Plasma lipidomic profiling method based on ultrasound extraction and liquid chromatography mass spectrometry. Anal Chem 2013;85:12085-12092. doi:10.1021/ac403181c.

[23] Castro-Perez JM, Kamphorst J, DeGroot J, Lafeber F, Goshawk J, Yu K, et al. Comprehensive LC-MS E lipidomic analysis using a shotgun approach and its application to biomarker detection and identification in osteoarthritis patients. J Proteome Res 2010;9:2377-2389. doi:10.1021/pr901094j.

[24] Jové M, Moreno-Navarrete JM, Pamplona R, Ricart W, Portero-Otín M, Fernández-Real JM. Human omental and subcutaneous adipose tissue exhibit specific lipidomic signatures. FASEB J 2014;28:1071-1081. doi:10.1096/fj.13234419.

[25] Sana TR, Roark JC, Li X, Waddell K, Fischer SM. Molecular formula and METLIN Personal Metabolite Database matching applied to the identification of compounds generated by LC/TOF-MS. J Biomol Tech 2008;19:258-266.

[26] Xia J, Sinelnikov IV, Han B, Wishart DS. MetaboAnalyst 3.0--making metabolomics more meaningful. Nucleic Acids Res 2015;43:W251-7. doi:10.1093/nar/gkv380.

[27] Kamburov A, Pentchev K, Galicka H, Wierling C, Lehrach H, Herwig R. ConsensusPathDB: toward a more complete picture of cell biology. Nucleic Acids Res 2011;39:D712-7. doi:10.1093/nar/gkq1156.

[28] Warde-Farley D, Donaldson SL, Comes O, Zuberi K, Badrawi R, Chao P, et al. The GeneMANIA prediction server: biological network integration for gene prioritization and predicting gene function. Nucleic Acids Res 2010;38:W214-20. doi:10.1093/nar/gkq537.

[29] White UA, Stephens JM. Transcriptional factors that promote formation of white adipose tissue. Mol Cell Endocrinol 2010;318:10-14. doi:10.1016/j.mce.2009.08.023.

[30] Spurlock ME, Gabler NK. The development of porcine models of obesity and the metabolic syndrome. J Nutr 2008;138:397-402. doi:10.1093/jn/138.2.397.

[31] Pawar AS, Zhu X-Y, Eirin A, Tang H, Jordan KL, Woollard JR, et al. Adipose tissue remodeling in a novel domestic porcine model of diet-induced obesity. Obesity (Silver Spring) 2015;23:399-407. doi:10.1002/oby.20971.

[32] Hill JO, Wyatt HR, Peters JC. Energy balance and obesity. Circulation 2012;126:126-132. doi:10.1161/CIRCULATIONAHA.111.087213.

[33] Steig AJ, Jackman MR, Giles ED, Higgins JA, Johnson GC, Mahan C, et al. Exercise reduces appetite and traffics excess nutrients away from energetically 
687

688

689

690

691

692

693

694

695

696

697

698

699

700

701

702

703

704

705

706

707

708

709

710

711

712

713

714

715

716

717

718

719

720

721

722

723

724

725

726

efficient pathways of lipid deposition during the early stages of weight regain. Am J Physiol Regul Integr Comp Physiol 2011;301:R656-67. doi:10.1152/ajpregu.00212.2011.

[34] Zhang X, Lerman LO. The metabolic syndrome and chronic kidney disease. Transl Res 2017;183:14-25. doi:10.1016/j.trsl.2016.12.004.

[35] Galili O, Versari D, Sattler KJ, Olson ML, Mannheim D, McConnell JP, et al. Early experimental obesity is associated with coronary endothelial dysfunction and oxidative stress. Am J Physiol Heart Circ Physiol 2007;292:H904-11. doi:10.1152/ajpheart.00628.2006.

[36] Wu G, Zhang L, Li T, Zuniga A, Lopaschuk GD, Li L, et al. Choline supplementation promotes hepatic insulin resistance in phosphatidylethanolamine $\mathrm{N}$-methyltransferase-deficient mice via increased glucagon action. J Biol Chem 2013;288:837-847. doi:10.1074/jbc.M112.415117.

[37] Jacobs RL, Zhao Y, Koonen DPY, Sletten T, Su B, Lingrell S, et al. Impaired de novo choline synthesis explains why phosphatidylethanolamine $\mathrm{N}$ methyltransferase-deficient mice are protected from diet-induced obesity. J Biol Chem 2010;285:22403-22413. doi:10.1074/jbc.M110.108514.

[38] Holčapek M, Ovčačíková M, Lísa M, Cífková E, Hájek T. Continuous comprehensive two-dimensional liquid chromatography-electrospray ionization mass spectrometry of complex lipidomic samples. Anal Bioanal Chem 2015;407:5033-5043. doi:10.1007/s00216-015-8528-2.

[39] Meikle PJ, Wong G, Barlow CK, Weir JM, Greeve MA, Maclntosh GL, et al. Plasma lipid profiling shows similar associations with prediabetes and type 2 diabetes. PLoS One 2013;8:e74341. doi:10.1371/journal.pone.0074341.

[40] Wallace M, Morris C, O'Grada CM, Ryan M, Dillon ET, Coleman E, et al. Relationship between the lipidome, inflammatory markers and insulin resistance. Mol Biosyst 2014;10:1586-1595. doi:10.1039/c3mb70529c.

[41] Heilbronn LK, Coster ACF, Campbell LV, Greenfield JR, Lange K, Christopher $\mathrm{MJ}$, et al. The effect of short-term overfeeding on serum lipids in healthy humans. Obesity (Silver Spring) 2013;21:E649-59. doi:10.1002/oby.20508.

[42] Douglass JD, Zhou YX, Wu A, Zadrogra JA, Gajda AM, Lackey Al, et al. Global deletion of MGL in mice delays lipid absorption and alters energy homeostasis and diet-induced obesity. J Lipid Res 2015;56:1153-1171. doi:10.1194/jlr.M058586.

[43] Wu X-F, Wu X-X, Guo W-J, Luo Q, Gu Y-H, Shen Y, et al. Cerebroside D, a glycoceramide compound, improves experimental colitis in mice with multiple targets against activated T lymphocytes. Toxicol Appl Pharmacol 2012;263:296302. doi:10.1016/j.taap.2012.07.001.

[44] Margalit M, Shalev Z, Pappo O, Sklair-Levy M, Alper R, Gomori M, et al. Glucocerebroside ameliorates the metabolic syndrome in OB/OB mice. J Pharmacol Exp Ther 2006;319:105-110. doi:10.1124/jpet.106.104950.

[45] Chavez JA, Summers SA. A ceramide-centric view of insulin resistance. Cell Metab 2012;15:585-594. doi:10.1016/j.cmet.2012.04.002.

[46] Eisinger K, Liebisch G, Schmitz G, Aslanidis C, Krautbauer S, Buechler C. Lipidomic analysis of serum from high fat diet induced obese mice. Int J Mol Sci 2014;15:2991-3002. doi:10.3390/ijms15022991.

[47] Weir JM, Wong G, Barlow CK, Greeve MA, Kowalczyk A, Almasy L, et al. Plasma lipid profiling in a large population-based cohort. J Lipid Res 2013;54:2898-2908. doi:10.1194/jlr.P035808.

[48] Korkes HA, Sass N, Moron AF, Câmara NOS, Bonetti T, Cerdeira AS, et al. Lipidomic assessment of plasma and placenta of women with early-onset preeclampsia. PLoS One 2014;9:e110747. doi:10.1371/journal.pone.0110747.

[49] Leventis PA, Grinstein S. The distribution and function of phosphatidylserine in cellular membranes. Annu Rev Biophys 2010;39:407-427. doi:10.1146/annurev.biophys.093008.131234. 
[50] Grace MS, Dempsey PC, Sethi P, Mundra PA, Mellett NA, Weir JM, et al. Breaking up prolonged sitting alters the postprandial plasma lipidomic profile of adults with type 2 diabetes. J Clin Endocrinol Metab 2017;102:1991-1999. doi:10.1210/jc.2016-3926.

[51] Sanyal AJ, Pacana T. A Lipidomic Readout of Disease Progression in A DietInduced Mouse Model of Nonalcoholic Fatty Liver Disease. Trans Am Clin Climatol Assoc 2015;126:271-288.

[52] Gorden DL, Myers DS, Ivanova PT, Fahy E, Maurya MR, Gupta S, et al. Biomarkers of NAFLD progression: a lipidomics approach to an epidemic. J Lipid Res 2015;56:722-736. doi:10.1194/jl.P056002.

[53] Aburasayn H, Al Batran R, Ussher JR. Targeting ceramide metabolism in obesity. Am J Physiol Endocrinol Metab 2016;311:E423-35. doi:10.1152/ajpendo.00133.2016.

[54] Giesbertz P, Daniel H. Branched-chain amino acids as biomarkers in diabetes. Curr Opin Clin Nutr Metab Care 2016;19:48-54. doi:10.1097/MCO.0000000000000235.

[55] Polakof S, Dardevet D, Lyan B, Mosoni L, Gatineau E, Martin J-F, et al. Time Course of Molecular and Metabolic Events in the Development of Insulin Resistance in Fructose-Fed Rats. J Proteome Res 2016;15:1862-1874. doi:10.1021/acs.jproteome.6b00043.

[56] Zhang J, Zhang Y, Sun T, Guo F, Huang S, Chandalia M, et al. Dietary obesityinduced Egr-1 in adipocytes facilitates energy storage via suppression of FOXC2. Sci Rep 2013;3:1476. doi:10.1038/srep01476.

[57] Aljada A, Ghanim H, Mohanty P, Kapur N, Dandona P. Insulin inhibits the proinflammatory transcription factor early growth response gene-1 (Egr)-1 expression in mononuclear cells (MNC) and reduces plasma tissue factor (TF) and plasminogen activator inhibitor-1 (PAl-1) concentrations. J Clin Endocrinol Metab 2002;87:1419-1422. doi:10.1210/jcem.87.3.8462.

[58] Giannini C, Feldstein AE, Santoro N, Kim G, Kursawe R, Pierpont B, et al. Circulating levels of FGF-21 in obese youth: associations with liver fat content and markers of liver damage. J Clin Endocrinol Metab 2013;98:2993-3000. doi:10.1210/jc.2013-1250.

[59] Liu P, Hsieh P, Lin H, Liu T, Wu H, Chen C, et al. Grail is involved in adipocyte differentiation and diet-induced obesity. Cell Death Dis 2018;9:525. doi:10.1038/s41419-018-0596-8.

[60] Son MJ, Kim WK, Oh K-J, Park A, Lee DS, Han BS, et al. Methyltransferase and demethylase profiling studies during brown adipocyte differentiation. BMB Rep 2016;49:388-393. doi:10.5483/BMBRep.2016.49.7.062.

[61] Wright TM, King MV, Davey WG, Langley-Evans SC, Voigt J-PW. Impact of cafeteria feeding during lactation in the rat on novel object discrimination in the offspring. Br J Nutr 2014;112:1-5. doi:10.1017/S0007114514003134.

[62] Krawczyńska A, Herman AP, Antushevich H, Bochenek J, Dziendzikowska K, Gajewska A, et al. Modifications of Western-type diet regarding protein, fat and sucrose levels as modulators of steroid metabolism and activity in liver. J Steroid Biochem Mol Biol 2017;165:331-341. doi:10.1016/j.jsbmb.2016.07.012.

[63] Sari G, Meester EJ, van der Zee LC, Wouters K, van Lennep JR, Peppelenbosch M, et al. A mouse model of humanized liver shows a human-like lipid profile, but does not form atherosclerotic plaque after western type diet. Biochem Biophys Res Commun 2020. doi:10.1016/j.bbrc.2020.01.067.

[64] Statovci D, Aguilera M, MacSharry J, Melgar S. The impact of western diet and nutrients on the microbiota and immune response at mucosal interfaces. Front Immunol 2017;8:838. doi:10.3389/fimmu.2017.00838.

[65] Sumner LW, Amberg A, Barrett D, Beale MH, Beger R, Daykin CA, et al. Proposed minimum reporting standards for chemical analysis Chemical Analysis 
Working Group (CAWG) Metabolomics Standards Initiative (MSI). Metabolomics 2007;3:211-221. doi:10.1007/s11306-007-0082-2.

[66] Vukovic R, Dos Santos TJ, Ybarra M, Atar M. Children with metabolically healthy obesity: A review. Front Endocrinol (Lausanne) 2019;10:865. doi:10.3389/fendo.2019.00865. 


\section{FIGURE LEGENDS}

Figure 1. A western-type diet induces prepubertal obesity, and it defines a specific lipidomic signature in plasma. A) Representative CT images of the porcine model of prepuberal obesity showing accumulation of AT in animals under a western-type diet. B) Example of determination of subcutaneous fat (red), intermuscular fat (green), and flare fat, including pelvicrenal depot (orange) from CT images. C) Hierarchical clustering and heatmap shows a plasma lipidomic signature of high-calorie (western-type diet) in the porcine model. In the right panel, the PLS-DA model using plasma lipidomics demonstrates high accuracy $\left(>65 \%, R^{2}=0.99\right)$ in classification. Selected lipids are significantly associated with individual calorie intake (D), global fat abundance $(E)$, and pelvic-rrenal fat abundance $(F)$ as measured by CT in vivo. Shown lipids are a selection from those showing a significant correlation with specific traits (at least $p<0.05$ by Spearman's rank correlation test, lines indicate non-linear fit (exponential growth equations; $\left.Y=Y 0^{*} \exp \left(k^{*} X\right)\right)$. see Supplemental Data for the whole list).

Figure 2. The prepubescent obesity model described induces changes in circulating insulin levels and its action, in association with specific plasma lipids and with changes in AT insulin-regulated proteins. Association of plasma concentration of selected lipids with individual insulin concentrations and HOMA-IR values (A). B shows a representative western-blot of insulin-responsive signals in AT and the influence of western-type diet. Right graphs after each blot show densitometric integrated values normalized to the intensity of animals fed with conventional diets all adjusted to total protein content, estimated by Coomassie blue staining, with $p$ values after Students t-test. Considering all values from each protein, with a two way ANOVA significant differences were found as well for Glut2 in the subcutaneous depot, and for SREBP1 in omental and pelvic-renal depots $(p<0.05, p<0.01$ and $p<0.02$ respectively by post hoc analyses, $n$ ranging 3 to 9 different samples). C) Subcutaneous adipose tissue leptin mRNA levels are associated with specific circulating lipids. D) Venn diagram showing the specificity of correlations, based on the very low (or absent) overlap between circulating lipids associated (at least $p<0.05$ by Spearman's rank correlation test) with HOMA-IR, with subcutaneous adipose tissue leptin mRNA, and with fat content. Shown lipids in $A$ and $C$ are a selection from those showing significant correlation with these specific traits (at least $p<0.05$ by Spearman's rank 
correlation test, lines indicate non-linear fit (exponential growth equations; $Y=Y 0^{*} \exp \left(k^{*} X\right)$, see

820 Supplemental Data for the whole list)

Figure 3. Liver insulin-responsive signals and relationship with circulating lipidome. A)

823 Representative western-blot of insulin-responsive signals in the liver with * in Glut 2 indicating differences induced by western-type diet ( $p<0.05$ by Student's t-test). B) Liver triacylglyceride (TAG) levels are associated with specific circulating lipids. C) Venn diagram showing the specificity of correlations, based on the very low (or absent) overlap between circulating lipids associated (at least $p<0.05$ by Spearman's rank correlation test) with hepatic TAG levels, with HOMA-IR, with subcutaneous adipose tissue leptin mRNA and with fat content. Shown lipids in $B$ are a selection from those showing significant correlation with specific traits (at least $p<0.05$ by Spearman's rank correlation test, lines indicate non-linear fit (exponential growth equations; $\left.\mathrm{Y}=\mathrm{Y} 0^{*} \exp \left(\mathrm{k}^{*} \mathrm{X}\right)\right)$, see Supplemental Data for the whole list)

833 Figure 4. Validation in humans with obesity of proposed biomarkers. Specific lipids show a 834 similar positive correlation with BMI (in humans, in B, D, and F) and AT abundance (in the porcine 835 model, in A, C, and E). The same is presented for HOMA-IR correlations (for pigs in G and 836 humans in $\mathrm{H}$ ). All correlations are significant (at least $\mathrm{p}<0.05$ ) by Spearman's rank correlation 837 test. Lines indicate non-linear fit (exponential growth equations; $Y=Y 0^{*} \exp \left(k^{*} X\right)$ ). Pig icon comes 838 from aLf; and Human one by Ma Qing both from the Noun Project (thenountproject.com)

840 Figure 5. Interactomics of plasma metabolites associated with a western-type diet.

841 Metabolites were mapped to a pathway database (in this case, KEGG), and nodes, representing 842 pathways (identified by pathway name in the database), is proportional to the number of 843 metabolites contained in the pathway. Node color intensity is associated with the hypergeometric 844 test accounting number of metabolites associated with high-calorie diet and those potentially 845 present in the specific pathway (node), while edge width represents the percentage of shared 846 metabolites between pathways and edge color indicate the number of metabolites associated 847 with western-type diet. 
849 Table 1. Morphological, calorie consumption and biochemical traits

\begin{tabular}{|c|c|c|c|}
\hline Parameter & Conventional diet & Western-type diet & $p^{1}$ \\
\hline Initial weight (Kg) & $16.5 \pm 2.8$ & $16.7 \pm 2.2$ & 0.86 \\
\hline Final weight $(\mathrm{Kg})$ & $53.2 \pm 5.7$ & $60 \pm 8.36$ & 0.043 \\
\hline $\begin{array}{l}\text { Weight gain } \\
(\mathrm{Kg} / \mathrm{d})\end{array}$ & $0.58 \pm 0.06$ & $0.68 \pm 0.1$ & 0.024 \\
\hline $\begin{array}{l}\text { Caloric } \\
\text { consumption } \\
\text { (Kcal/d) }\end{array}$ & $3819.7 \pm 236.5$ & $5213.9 \pm 516.8$ & $<0.0001$ \\
\hline $\begin{array}{l}\text { Fecal fat content } \\
(\mathrm{mg} / \mathrm{g})\end{array}$ & $19.5 \pm 1.3$ & $50 \pm 8$ & 0.02 \\
\hline $\begin{array}{l}\text { Pelvico-renal fat } \\
\text { weight }(\mathrm{Kg})\end{array}$ & $0.188 \pm 0.026$ & $0.405 \pm 0.082$ & 0.008 \\
\hline $\begin{array}{l}\text { Relative fat } \\
\text { volume at VL2 } \\
(\%)\end{array}$ & $12.7 \pm 1.2$ & $19.4 \pm 2.6$ & 0.002 \\
\hline $\begin{array}{l}\text { Blood Cholesterol } \\
\text { (mg/dL) }\end{array}$ & $125.9 \pm 24.4$ & $155.7 \pm 26.4$ & 0.015 \\
\hline $\begin{array}{l}\text { Blood LDL- } \\
\text { Cholesterol } \\
\text { (mg/dL) }\end{array}$ & $46.8 \pm 8.1$ & $57.1 \pm 8.5$ & 0.011 \\
\hline $\begin{array}{l}\text { Blood HDL- } \\
\text { Cholesterol } \\
\text { (mg/dL) }\end{array}$ & $45.2 \pm 9.9$ & $61.3 \pm 13.8$ & 0.007 \\
\hline $\begin{array}{l}\text { Triacylglyceridemi } \\
\text { a } \\
(\mathrm{mg} / \mathrm{dL})\end{array}$ & $21.6 \pm 6.3$ & $29.2 \pm 5.9$ & 0.014 \\
\hline $\begin{array}{l}\text { Glycemia } \\
(\mathrm{mg} / \mathrm{dL})\end{array}$ & $80.75 \pm 16.73$ & $108.6 \pm 23.9$ & 0.01 \\
\hline $\begin{array}{l}\text { Fructosamine } \\
\text { (mg/dL) }\end{array}$ & $177.6 \pm 24.9$ & $152.1 \pm 46.8$ & 0.131 \\
\hline $\begin{array}{l}\text { Insulinemia } \\
(\mathrm{pg} / \mathrm{mL})\end{array}$ & $5.65 \pm 0.24$ & $5.77 \pm 0.46$ & 0.26 \\
\hline HOMA-IR & $1.09 \pm 0.21$ & $1.56 \pm 0.39$ & 0.009 \\
\hline
\end{tabular}

850 Values shown are mean $\pm S D$, unless stated otherwhise. ${ }^{1}$ Student's $t$ test 851 comparison between values in in conventional and western-type diets 


\section{FIGURE 1}

A

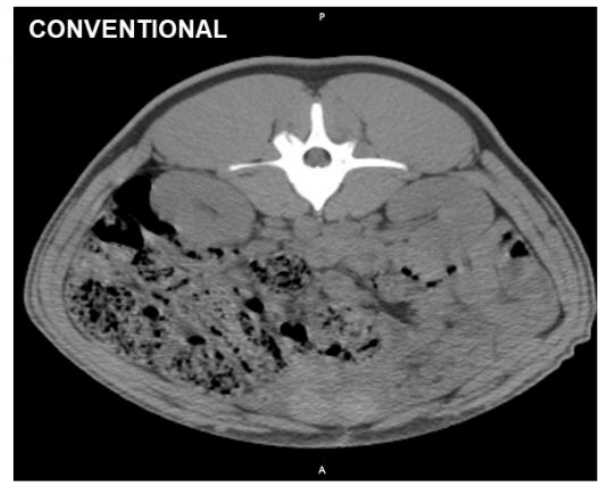

C

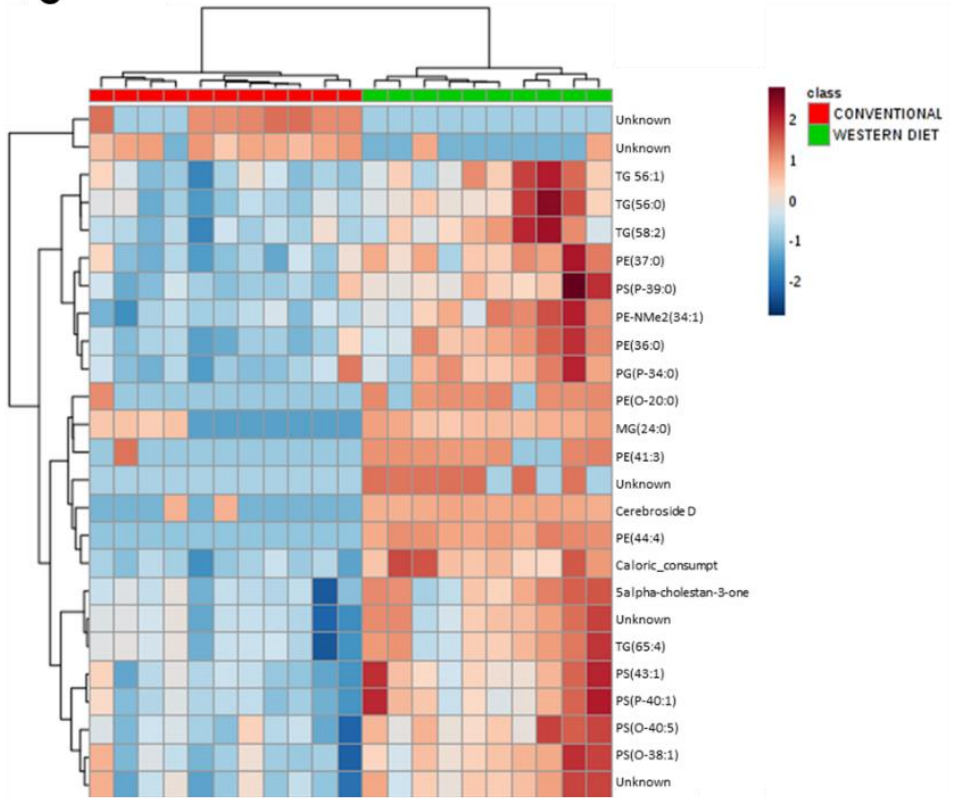

D

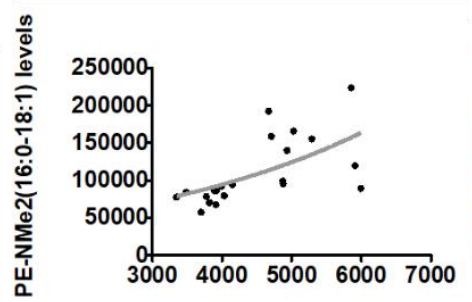

Calorie intake

E

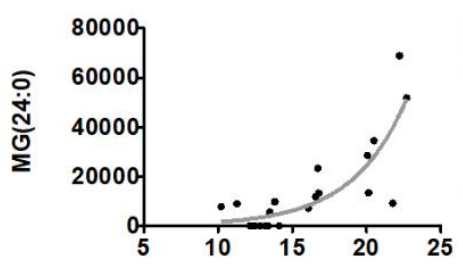

Fat abundance

( $\%$ body weight)

F

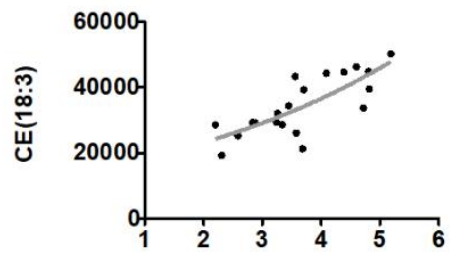

Pelvicorrenal fat abundance ( $\%$ body weight)

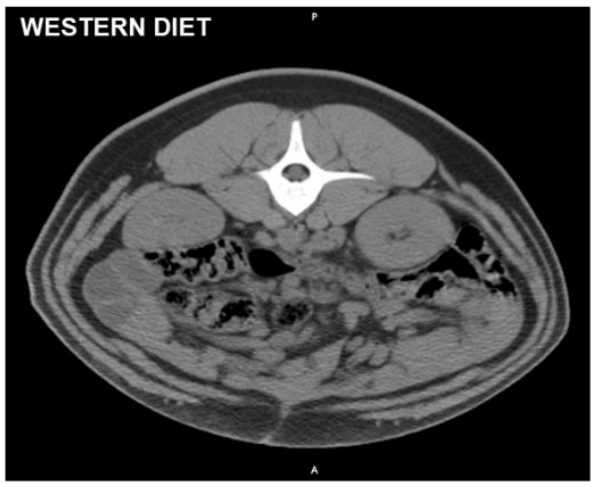

B
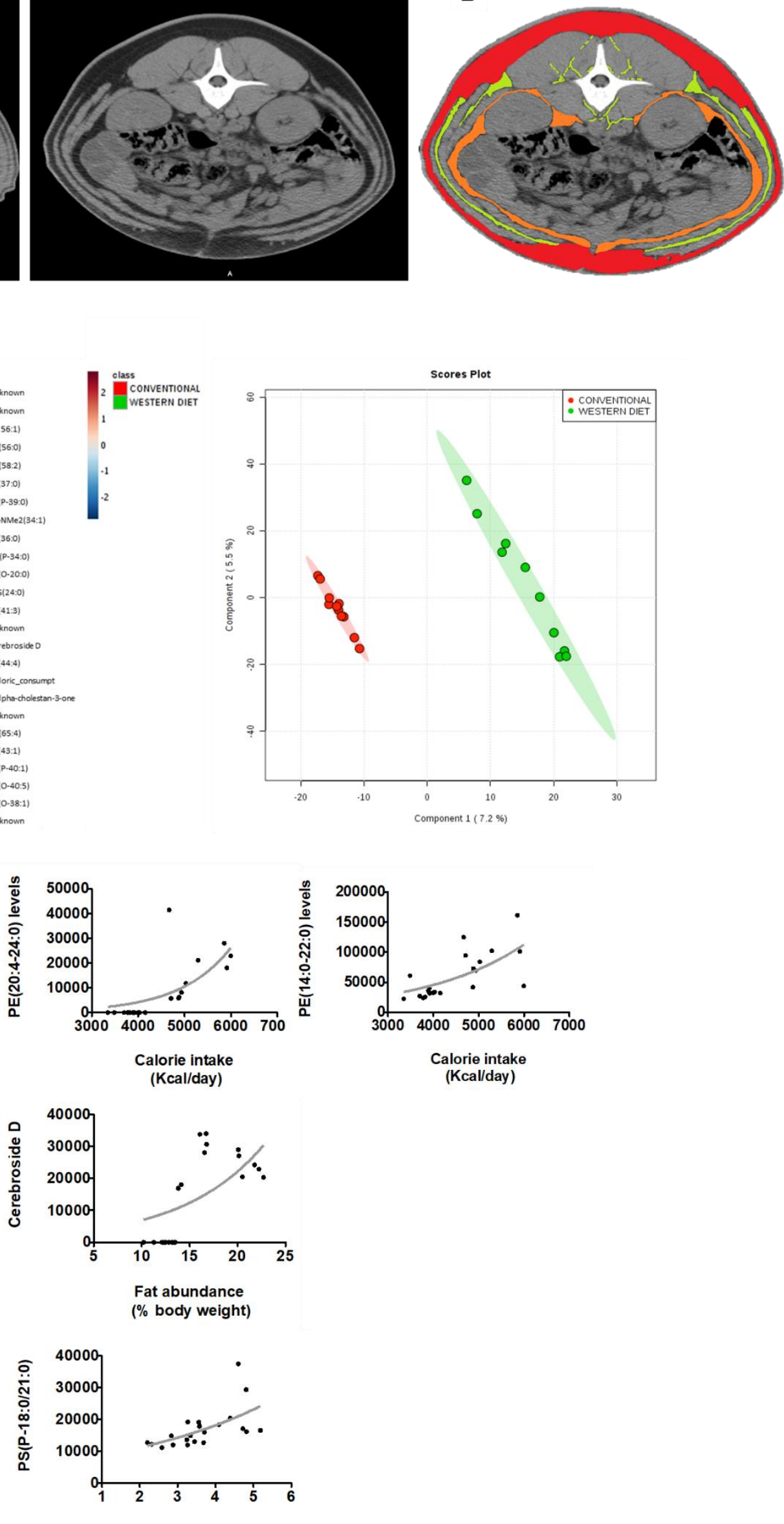

Pelvicorrenal fat abundance ( $\%$ body weight) 
FIGURE 2

A
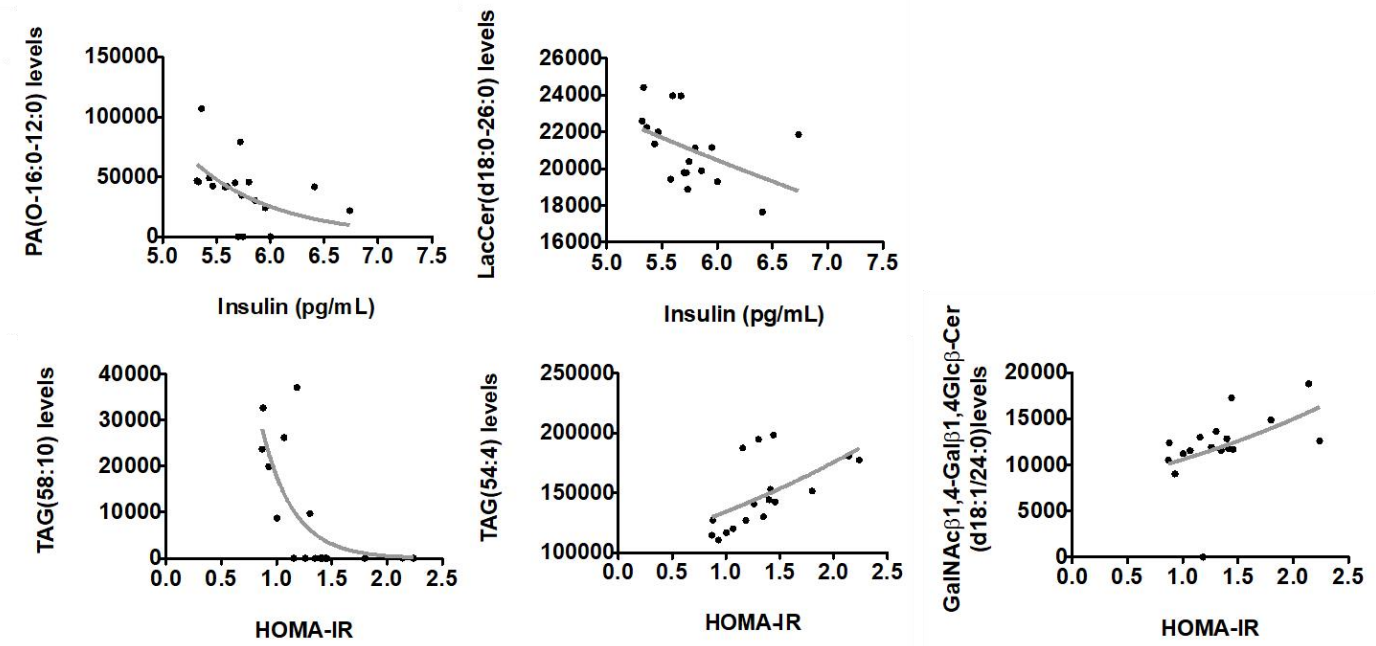

B

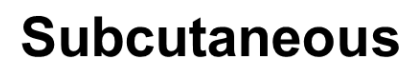

Omental

\section{Pelvicorenal}

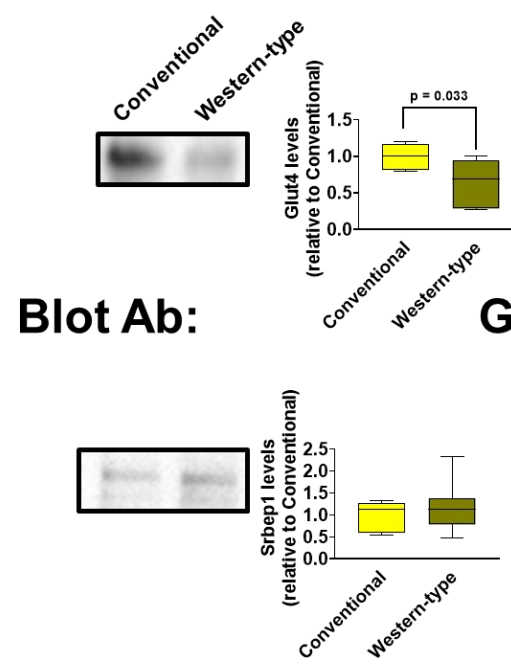

Blot Ab:
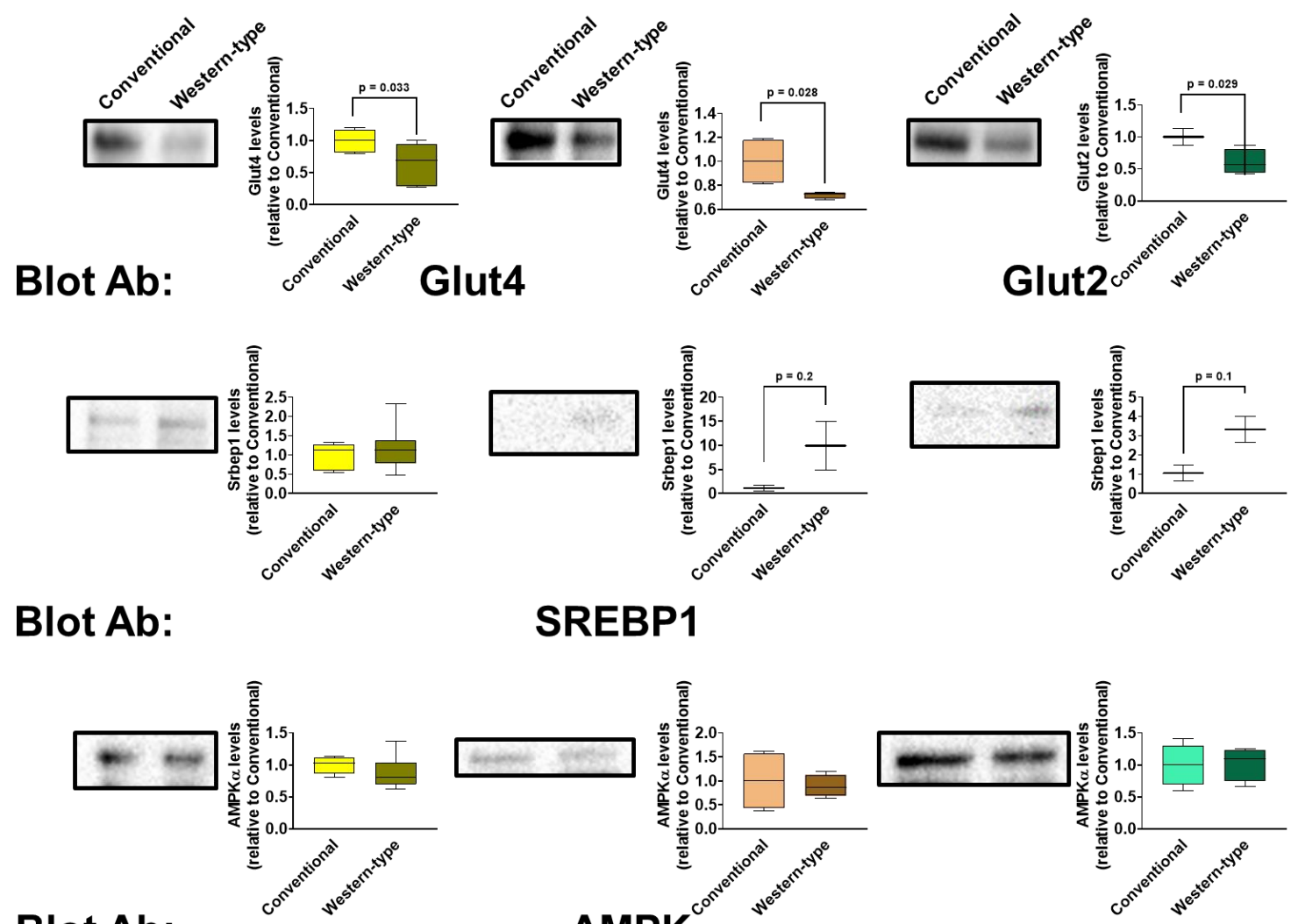

Blot Ab:
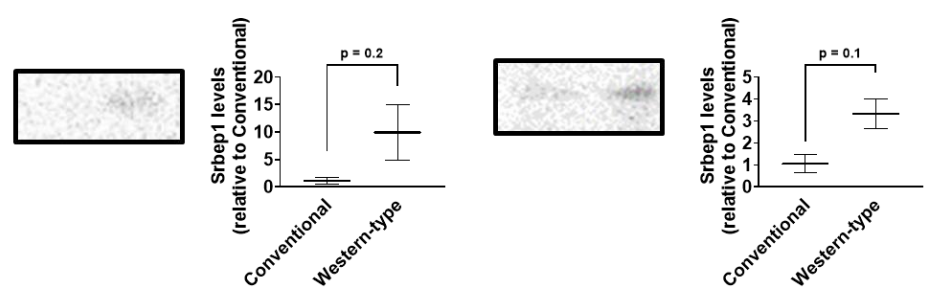

SREBP1
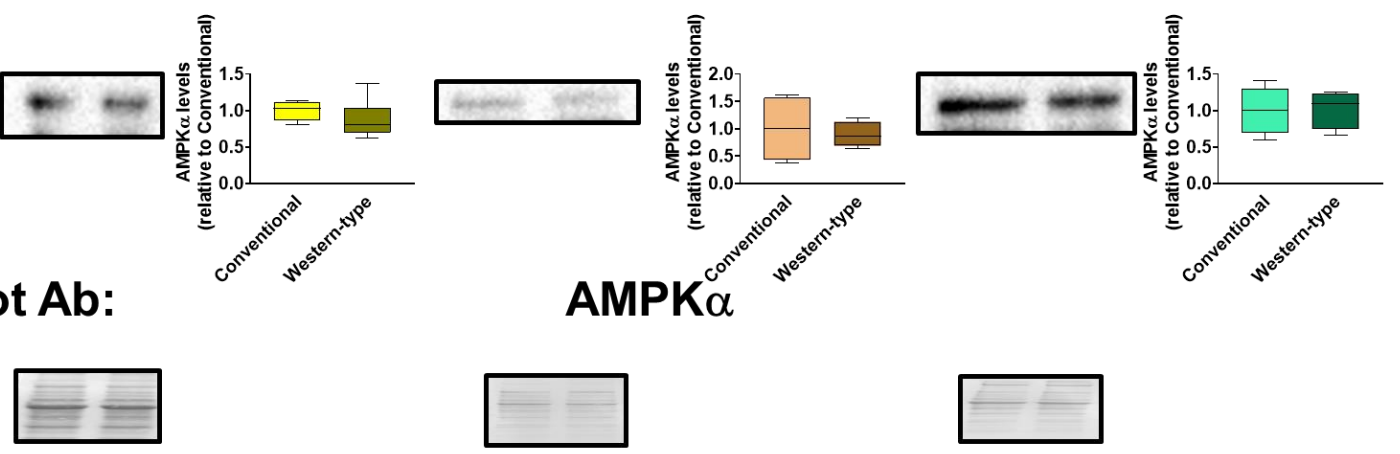

\section{Coomassie Blue}

C
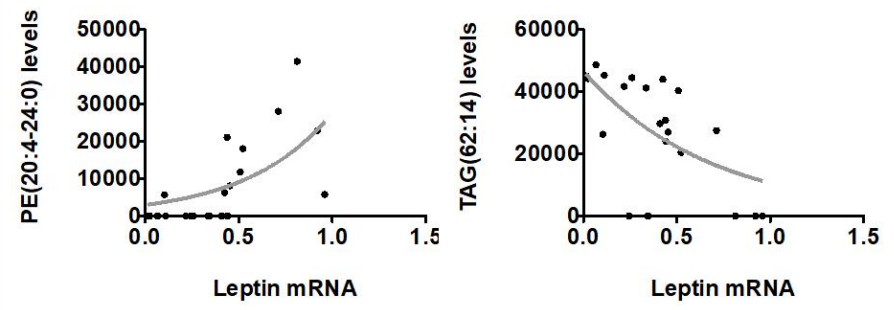

D HOMA-IR

SC Leptin mRNA

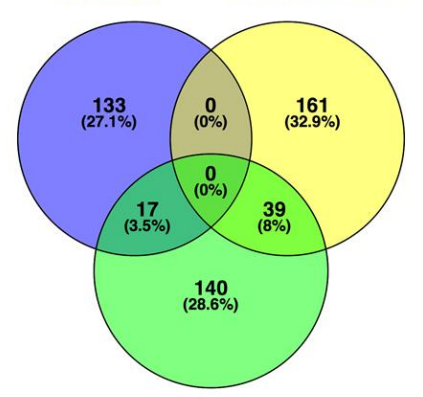




\section{FIGURE 3}

A

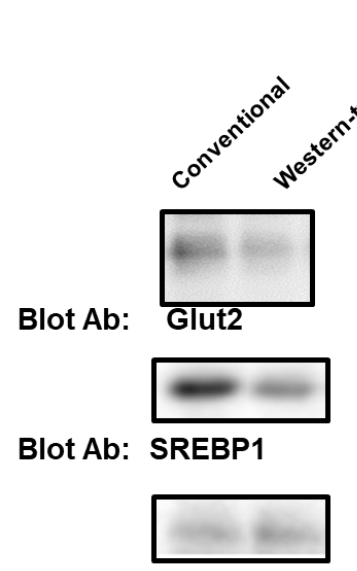

Blot Ab: AMPK $\alpha$
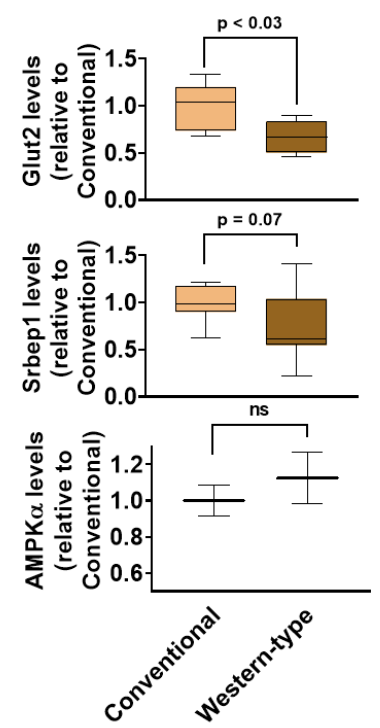

C

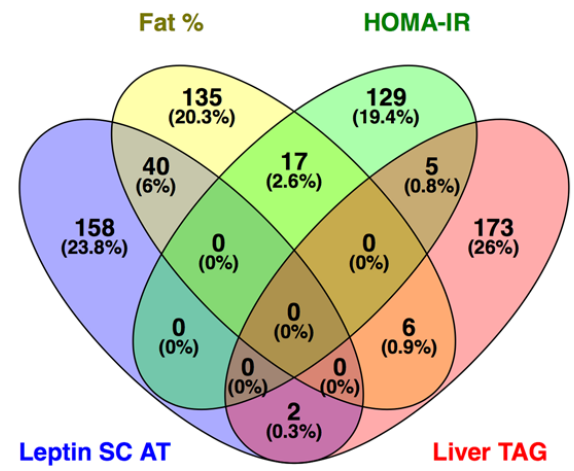

B

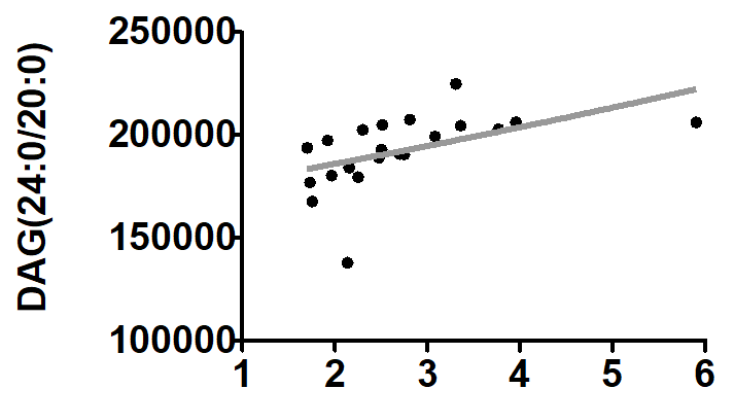

Liver TAG amount

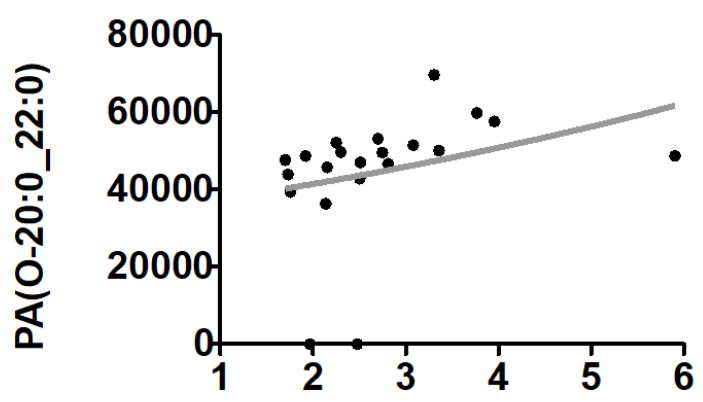

Liver TAG amount 
A

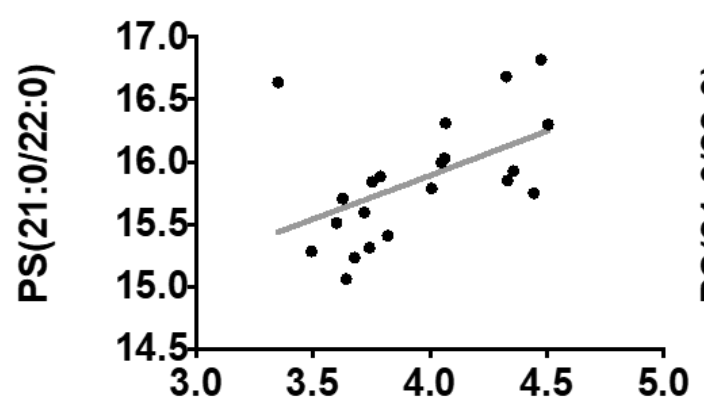

C

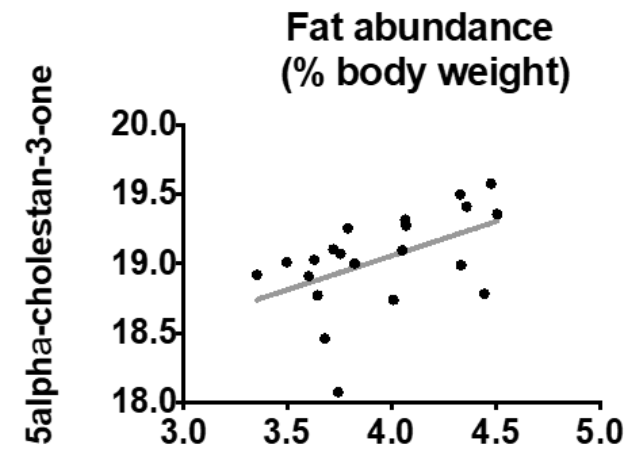

Fat abundance

E

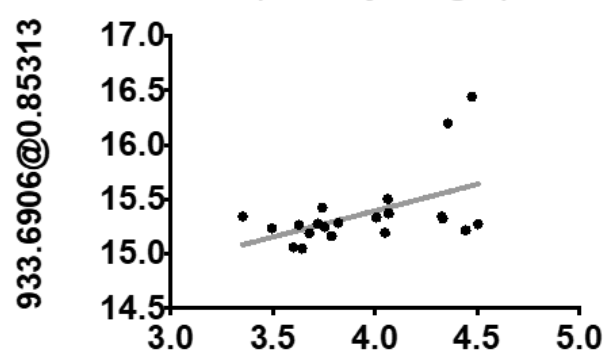

G

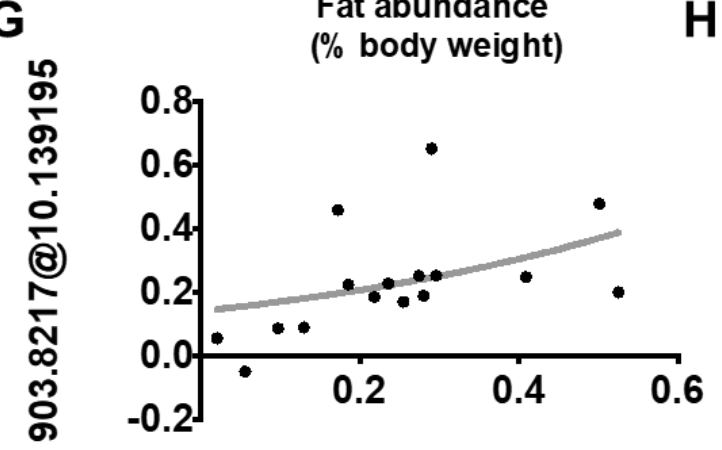

HOMA-IR

B

D

F

H
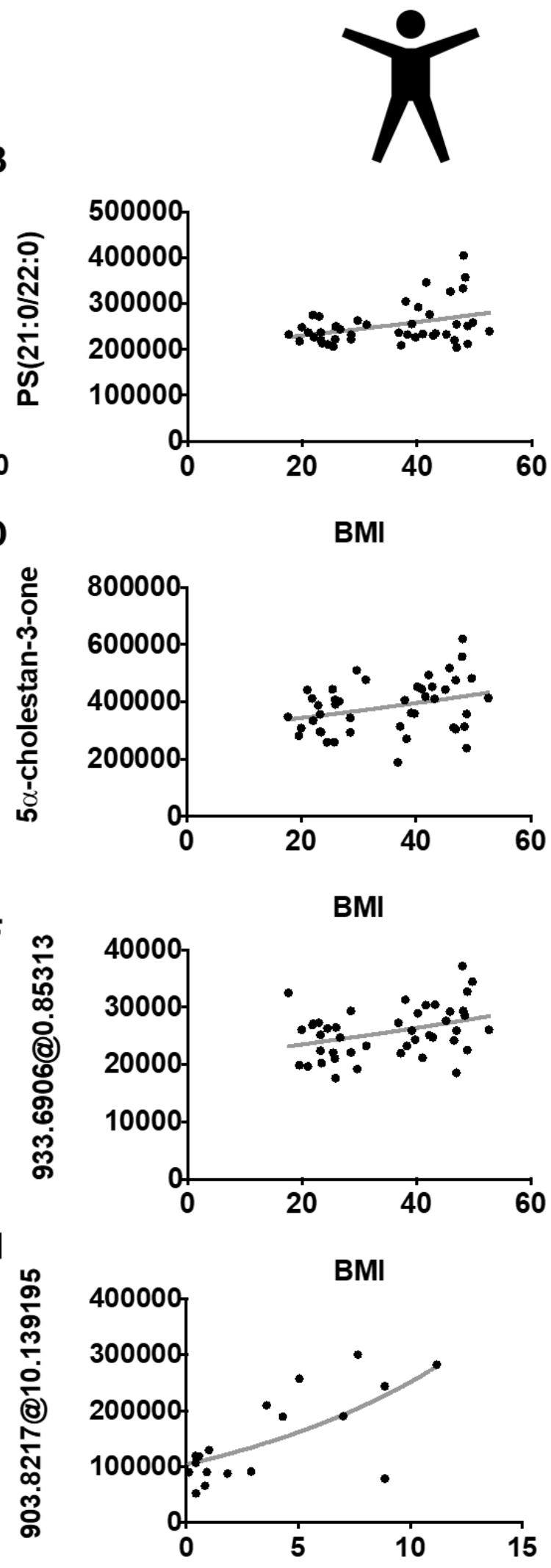

HOMA-IR 


\section{FIGURE 5}

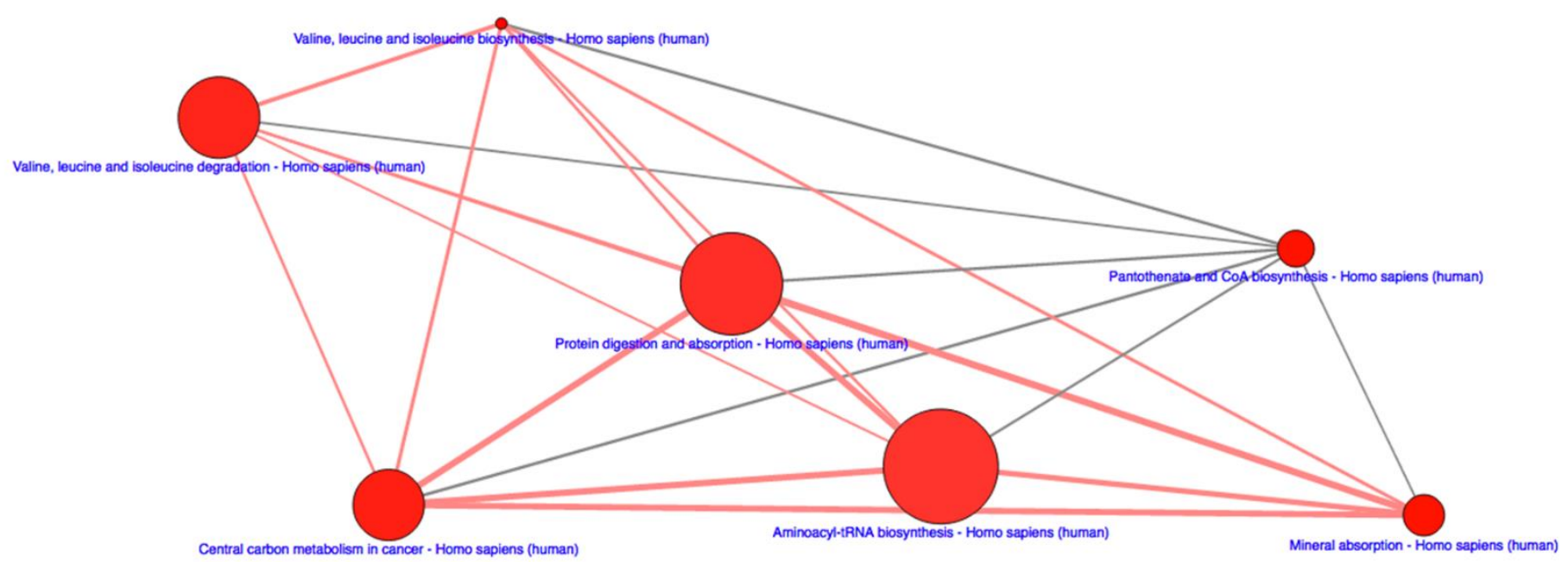
Node size (\# metabolites)

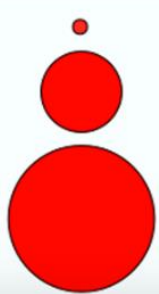
23 metabolites
34 metabolites

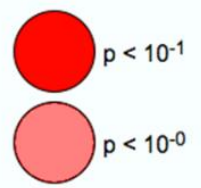
$-1 \%$
$=1$
$-50 \%$
$=0$
52 metabolites
$p=1.0$
$100 \%$
0

Edge color (metabolites from input) 


\section{EXTENDED METHODS}

\section{Chemicals}

3 For lipidomic analyses and other analytical procedures, synthetic lipids were obtained from 4 Avanti Polar Lipids Inc. (Alabaster, AL, USA) and Sigma-Aldrich (Madrid, Spain). Methyl tertbutyl ether (MTBE), acetonitrile, potassium chloride, chloroform, ammonium formate and ammonium hydroxide -all liquid chromatography-mass spectrometry (LC-MS) grade- were purchased from Sigma-Aldrich (Madrid, Spain); methanol was from Carlo Erba (Milano, Italy); acetone was from Riedel-de-Häen (Seelze, Germany); and LC/MS-grade isopropanol and formic acid were from Baker (Phillipsburg, NJ, USA).

\section{Computed tomography scanning and image analysis}

Previously to the scan, animals were fasted for $16 \mathrm{~h}$ and anesthetized with azaperone $(0.1$ $\mathrm{mg} / \mathrm{kg}$ body weight $(\mathrm{BW}))$, ketamine $(0.2 \mathrm{mg} / \mathrm{kg} \mathrm{BW})$, and, if necessary, propofol $(0.22 \mathrm{mg} / \mathrm{kg}$ BW) as explained in [1]. Pigs were scanned in the prone position, and acquisition parameters were axial, $1 \mathrm{~s}, 140 \mathrm{kV}, 145 \mathrm{~mA}, 5 \mathrm{~mm}$ thickness, matrix $512 \times 512$, and displayed field of view adapted to the size of each pig. Scanning lasted approximately 20 min per pig. For each pig, one axial image was obtained at the level of the $2^{\text {nd }}$ lumbar vertebrae $(2 \mathrm{VL})$.

Images were analyzed with the software VisualPork [2,3] developed by the University of Girona and IRTA. Image analysis was those explained in ValLaillet et al. [4] modified. Thus, the mean and standard deviation of the Hounsfield $(\mathrm{HU})$ value of the subcutaneous fat were obtained from each image. These values were used for the determination of the fat tissue content. From each image, an ROI (region of interest) was determined that considered all the outlines of the pig (ROI1), i.e., the total area of the image. Another area was taken at for the interior part of the subcutaneous fat of each image (ROI2), which corresponds to the area of the image, excluding subcutaneous fat and skin. Another area was obtained, including all the intraabdominal region, being the ribs, and the tenderloin the borders (ROI3). Finally, intraperitoneal area, going through the junction between flare fat and internal organs (ROI4), 
was determined. For each ROI (from ROI1 to ROI4), the AT area was calculated, considering the range of $\mathrm{HU}$ values between the mean $\mathrm{HU}$ value plus/minus two standard deviations previously determined. These areas were transformed into $\mathrm{mm}^{3}$, considering the DFOV, the matrix size, and the thickness [5]. From the volume of ROl1 minus those from ROI2, the subcutaneous fat was obtained. Intermuscular fat was obtained as the fat volume in ROI2 minus ROI3 and flare fat as the fat volume of ROI3 minus those of ROI4. The ratio between the volume of the total fat (subcutaneous, intermuscular, and flare fat) and the total volume of the image was determined and converted into a percentage (relative fat volume at VL2).

Samples were homogenized in a buffer containing $180 \mathrm{mM} \mathrm{KCl}$, five $\mathrm{mM}$ MOPS, two $\mathrm{mM}$ EDTA, one mM diethylenetriaminepentaacetic acid, and one $\mu \mathrm{M}$ butylated hydroxyl toluene, $10 \mu \mathrm{g} / \mathrm{ml}$ aprotinin, one $\mathrm{mM} \mathrm{NaF}$, one $\mathrm{mM} \mathrm{Na3VO}$ and a protease inhibitor mix (GE Healthcare $80-6501-23$, USA) $(1 \% \mathrm{v} / \mathrm{v})$ with a Potter-Elvehjem device, at $4{ }^{\circ} \mathrm{C}$. After brief centrifugation $(500 \times \mathrm{g}, 5 \mathrm{~min})$ protein concentrations were measured in the supernatants using the Bradford protein assay (BioRad Laboratories, München, Germany). Tissue protein (15-40 mg) was resolved by SDS-PAGE and electroblotted onto polyvinylidene difluoride membranes (Immobilon-P, Millipore, Bedford, USA). Immunodetection was performed using as primary and secondary antibodies, those listed in Supplemental Table 6. A monoclonal antibody to $\beta$-actin (Sigma, USA) and Coomassie blue staining were used to control protein loading. Protein bands were visualized with the chemiluminescence ECL method (Millipore Corporation, Billerica, MA, USA). Luminescence was recorded and quantified in Lumi-Imager equipment from Boehringer (Mannheim, Germany), using the Quantity One 4.6.5. Software.

\section{Lipidomic analyses}

Preparation of Lipid Standards Lipid standards consisting of isotopically labeled lipids (see Supplemental Table 7) were used for external standardization (i.e., lipid family assignment) and internal standardization (i.e., for adjustment of potential inter- and intra-assay variances). Stock solutions were prepared by dissolving lipid standards in MTBE at a concentration of $1 \mathrm{mg} / \mathrm{mL}$, and working solutions were diluted to $2.5 \mu \mathrm{g} / \mathrm{mL}$ in MTBE. 
Lipid extraction Briefly, in order to precipitate plasma protein fraction, $5 \mu$ of Mili $Q$ water and $20 \mu \mathrm{l}$ of methanol were added to $10 \mu \mathrm{l}$ of a plasma sample. After the addition, samples were

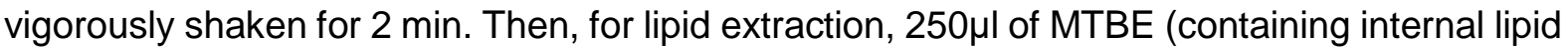
standards) were added, and samples were immersed in a water bath (ATU Ultrasonidos, Valencia, Spain) with an ultrasound frequency and power of $40 \mathrm{kHz}$ and $100 \mathrm{~W}$, respectively, at $10^{\circ} \mathrm{C}$ for $30 \mathrm{~min}$. Then, $75 \mu \mathrm{L}$ of Mili $\mathrm{Q}$ water was added to the mixture, and the organic phase was separated by centrifugation $(1,400 \times \mathrm{g})$ at $10^{\circ} \mathrm{C}$ for $10 \mathrm{~min}$. Lipid extracts contained in the upper phase were collected and subjected to mass spectrometry. A pool of all lipid extracts was prepared and used as quality controls, as previously described [6].

LC-MS/MS method Sample compartment was refrigerated at $4^{\circ} \mathrm{C}$, and for each sample, $10 \mu \mathrm{l}$ of lipid extract was applied onto $1.8 \mu \mathrm{m}$ particle $100 \times 2.1 \mathrm{~mm}$ id Waters Acquity HSS T3 column (Waters, Milford, MA, USA) heated to $55^{\circ} \mathrm{C}$. The flow rate was $400 \mu \mathrm{l} / \mathrm{min}$ with solvent A composed of $10 \mathrm{mM}$ ammonium acetate in acetonitrile-water $(40: 60, \mathrm{v} / \mathrm{v})$ and solvent B composed of $10 \mathrm{mM}$ ammonium acetate in acetonitrile-isopropanol $(10: 90, \mathrm{v} / \mathrm{v})$. The gradient started at $40 \%$ B and reached $100 \%$ B in 10 min and held for 2 min. Finally, the system was switched back to $60 \%$ B and equilibrated for $3 \mathrm{~min}$. Duplicate runs of the samples were performed to collect positive and negative electrospray ionized lipid species in a TOF mode, operated in full-scan mode at 100 to $3000 \mathrm{~m} / \mathrm{z}$ in an extended dynamic range (2 GHz), using $\mathrm{N}_{2}$ as nebulizer gas $\left(5 \mathrm{~L} / \mathrm{min}, 350^{\circ} \mathrm{C}\right)$. The capillary voltage was set $3500 \mathrm{~V}$ with a scan rate of 1scan/s. Continuous infusion using a double spray with masses 121.050873, 922.009798 (positive ion mode), and 119.036320, 966.000725 (negative ion mode) was used for in-run calibration of the mass spectrometer.

\section{Untargeted metabolomics analyses of serum}

For extraction of metabolites, $1.8 \mathrm{ml}$ of a methanol/water $8: 1(\mathrm{v} / \mathrm{v})$ was added to $200 \mu \mathrm{l}$ of serum sample. After stirring for $1 \mathrm{~min}$ and centrifugation $(1400 \mathrm{xg})$ for 10 min at $4^{\circ} \mathrm{C}$, the supernatant with water-soluble metabolites was placed into a new vial, and the pellet was washed twice 
with additional methanol/water. The combined upper-phases solutions were partially dried under $\mathrm{N}_{2}$ flow until methanol removal. Finally, the solution was quickly frozen and lyophilized.

The LC-MS analysis of aqueous serum extract samples was done using a Q-TOF/MS 6550 mass spectrometer (Agilent Technologies) with an Agilent UPLC 1290 chromatographic system. The lyophilized samples were reconstituted by adding $200 \mu \mathrm{l}$ of $0.1 \%$ formic acid in water and injected onto a $1.8 \mu \mathrm{m}$ particle $50 \times 2.1 \mathrm{~mm}$ id Zorbax SB-Aq RR column (Agilent Technologies) which was heated to $60^{\circ} \mathrm{C}$ in the column oven. A binary gradient system consisting of $0.2 \%$ acetic acid in water (solvent $\mathrm{A}$ ) and $0.2 \%$ acetic acid in methanol (solvent B) was used. The flow rate was $0.5 \mathrm{~mL} / \mathrm{min}$, and the solvent gradient program was 0 to $1 \mathrm{~min}$ $2 \% \mathrm{~B}$ isocratic, 1 to $13 \mathrm{~min} 98 \% \mathrm{~B}, 13$ to $19 \mathrm{~min} 98 \% \mathrm{~B}$ isocratic, $1 \mathrm{~min} 0 \% \mathrm{~B}$, and then equilibrated for an additional $5 \mathrm{~min}$. The autosampler temperature was maintained at $4^{\circ} \mathrm{C}$, and the injection volume was $10 \mu \mathrm{l}$.

Ionization was performed in an electrospray source mode, with a drying gas temperature and a flow of $150^{\circ} \mathrm{C}$ and $14 \mathrm{~mL} / \mathrm{min}$, respectively; a sheath gas temperature and flow of $300^{\circ} \mathrm{C}$ and $11 \mathrm{~mL} / \mathrm{min}$, respectively; a nebulizer pressure of $30 \mathrm{psi}$; a capillary voltage of $3,000 \mathrm{~V}$; and a nozzle voltage of 500 V. QTOF was operated in MS Full Scan mode in positive polarity, applying a fragmentary voltage of $380 \mathrm{~V}$, an acquisition rate of 5 spectra/s and an acquisition range from 50 to $1700 \mathrm{~m} / \mathrm{z}$. Blank samples and quality control samples were injected regularly between the runs to ensure the quality of the data.

\section{Peripheral blood monocyte cells (PBMC) and AT transcriptomics}

Briefly, PBMCs were isolated from animal blood by Ficoll gradient separation (GE Healthcare, Piscataway, NJ, USA) and PBMC total RNA was extracted using Tripure Reagent (Roche Diagnostic, Barcelona, Spain) and purified with Qiagen RNeasy Mini Kit spin columns (Izasa, Barcelona, Spain). Blood samples (10 mL) were diluted (1:1) with phosphate buffer saline (pH: 7.4), and PBMC were isolated by Ficoll gradient separation, according to the instructions indicated by the manufacturer (GE Healthcare Bio Sciences). The PBMC pellet was carefully 
resuspended with $4 \mathrm{~mL}$ of erythrocyte lysis buffer $\left(155 \mathrm{mM} \mathrm{NH}_{4} \mathrm{Cl}\right.$, ten $\mathrm{mM} \mathrm{KHCO}_{3}$, and 100

$111 \mu \mathrm{M}$ EDTA, pH: 7.4), incubated at room temperature for 5 minutes and centrifuged at $400 \mathrm{~g}$ for

11210 minutes. Finally, the supernatant was aspirated, and the PBMC pellet was stored at $-70^{\circ} \mathrm{C}$

113 until RNA analysis. PBMC total RNA was extracted using Tripure Reagent (Roche Diagnostic)

114 and purified with Qiagen RNeasy Mini Kit spin columns (Izasa). RNA quantity was measured

115 spectrophotometrically (NanoDrop $\left.{ }^{\circledR}\right)$, and RNA quality was confirmed with the Agilent 2100

116 Bioanalyzer (Agilent Technologies).

118 The gene expression levels were assessed using Porcine (V2) Gene Expression Microarray,

$1194 \times 44 \mathrm{~K}$ (Agilent Technologies) following the manufacturer's instructions. This microarray 120 quantifies 43,603 probe sets. Labeling and hybridization of the samples were performed 121 following Agilent One-color Microarray-Based Gene Expression Analysis-Low Input Quick

122 Amp Labelling kit v6.6 protocol. Arrays were scanned with a G2565A Microarray Scanner 123 System with SureScan High-Resolution Technology, and spot intensities were quantified 124 using Feature Extraction 11.5.1.1 (Agilent Technologies, Inc. Santa Clara, CA). Background 125 correction and inter-array normalization were performed with GeneSpring software v12.6.1

126 (Agilent Technologies). Gene expression summaries were created for each gene by averaging 127 all probe sets for each gene. All data analysis was conducted on gene-summarized data. Processed data were subsequently filtered for significant detection ( $P$-value $\leq 0.01)$.

130 Quantitative analyses of selected mRNA in subcutaneous AT was performed as previously 131 described [7]. Briefly, RNA was prepared from tissue samples using an RNeasy Lipid Tissue 132 Mini Kit (Izasa). The integrity of each RNA sample was checked by an Agilent Bioanalyzer 133 (Agilent Technologies). Total RNA was quantified by the use of a spectrophotometer 134 (GeneQuant; GE Healthcare) and reverse transcribed to cDNA using a High Capacity cDNA 135 Archive Kit (Applied Biosystems Inc., Madrid, Spain) according to the manufacturer's protocol. 136 Gene expression was assessed by real-time PCR using a LightCycler 480 Real-Time PCR 
137 System (Roche Diagnostics) using TaqMan® technology suitable for relative gene expression 138 quantification. 


\section{REFERENCES}

141 [1] Carabús A, Gispert M, Brun A, Rodríguez P, Font-i-Furnols M. In vivo computed tomography evaluation of the composition of the carcass and main cuts of growing pigs of three commercial crossbreeds. Livest Sci 2014;170:181-192. DOI:10.1016/j.livsci.2014.10.005.

[2] Boada I, Spinola J, Rodriguez J, Martínez R. VisualPork towards the simulation of a virtual butcher. II Workshop on the Use Of ... 2009.

[3] Bardera A, Martínez R, Boada I, Font-i-Furnols M. VisualPork towards the simulation of a virtual butcher. FAIM I Conference of COST ... 2012.

[4] Val-Laillet D, Blat S, Louveau I, Malbert CH. A computed tomography scan application to evaluate adiposity in a minipig model of human obesity. Br J Nutr 2010;104:17191728. DOI:10.1017/S0007114510002667.

[5] Furnols MF I, Teran MF, Gispert M. Estimation of lean meat content in pig carcasses using X-ray computed tomography and PLS regression. ... and Intelligent Laboratory ... 2009.

[6] Want EJ, Masson P, Michopoulos F, Wilson ID, Theodoridis G, Plumb RS, et al. Global metabolic profiling of animal and human tissues via UPLC-MS. Nat Protoc 2013;8:1732. DOI:10.1038/nprot.2012.135.

[7] Jové M, Moreno-Navarrete JM, Pamplona R, Ricart W, Portero-Otín M, FernándezReal JM. Human omental and subcutaneous adipose tissue exhibit specific lipidomic signatures. FASEB J 2014;28:1071-1081. DOI:10.1096/fj.13-234419. 


\section{Supplemental Figure 1}

A

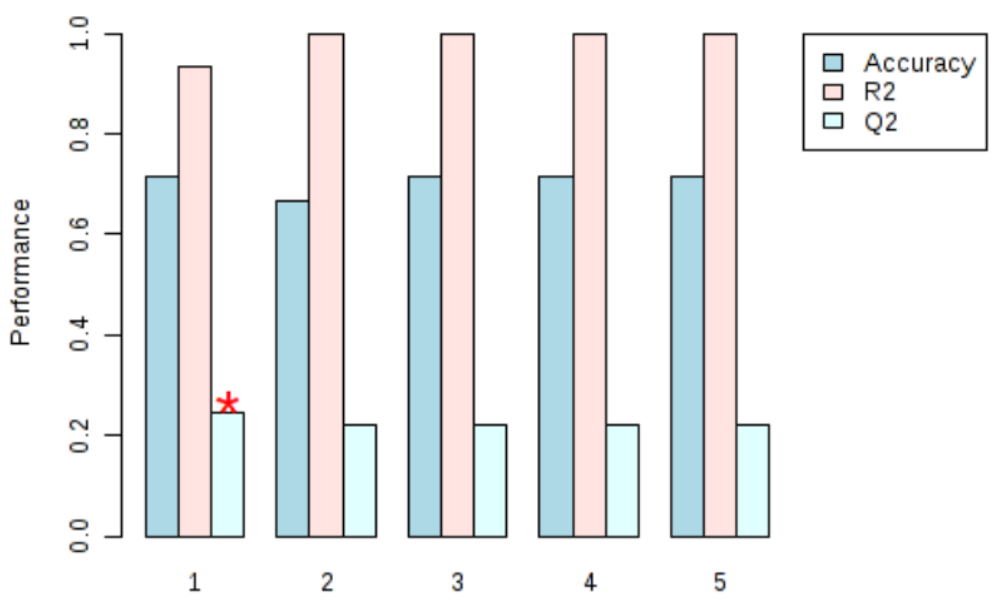

B

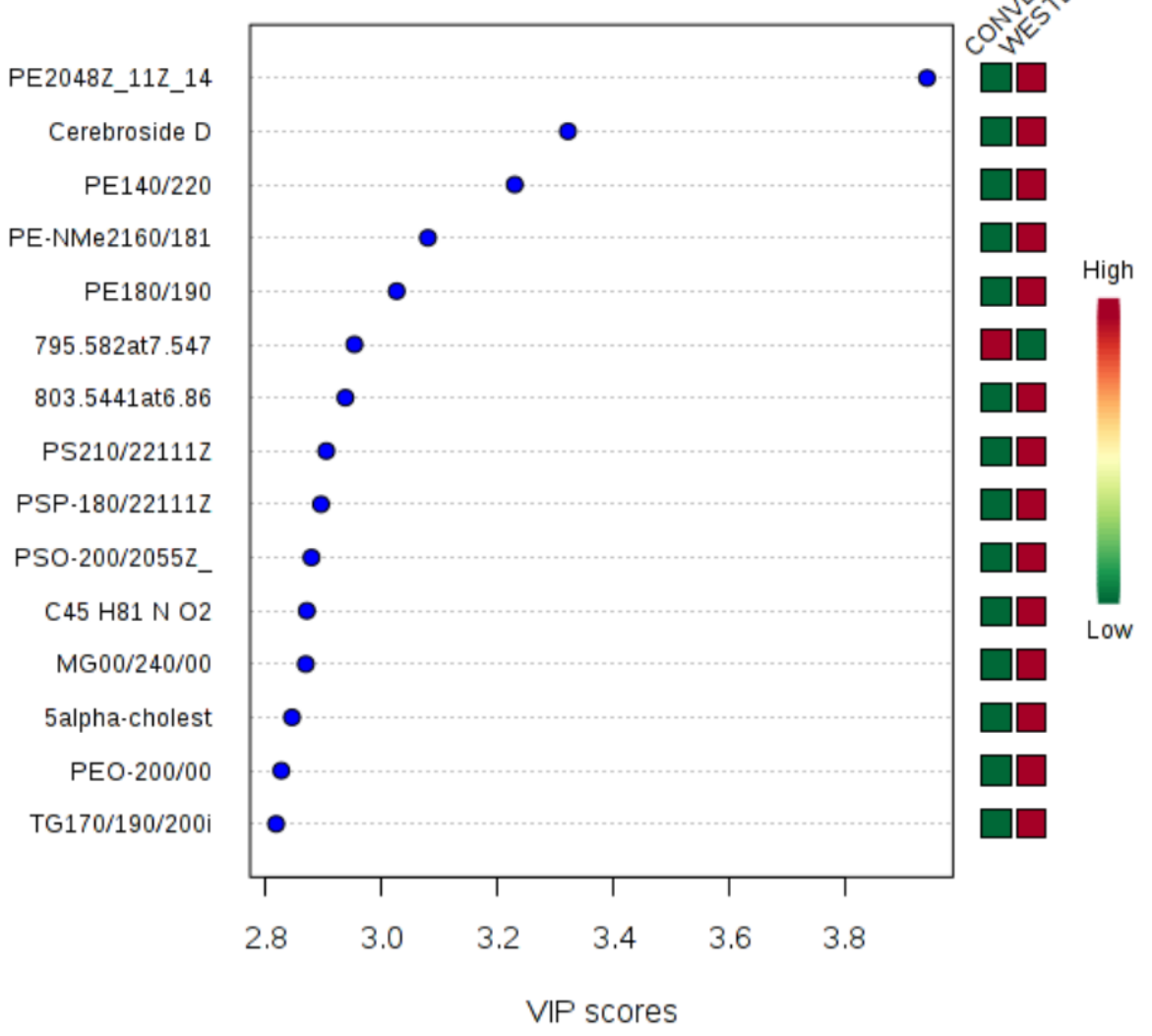


165 Supplemental Figure 1. Robustness of the PLS-DA model defining changes induced by a

166 western-type diet (Figure 1 in the main text) is ensured by high accuracy, R2, and Q2 values.

167 Variable importance in the projection of the first component demonstrates the importance of 168 specific lipid molecules in plasma in helping to define a lipidomic signature associated with 169 diet-induced obesity in prepubertal female pigs.

\section{Supplemental Figure 2}

A

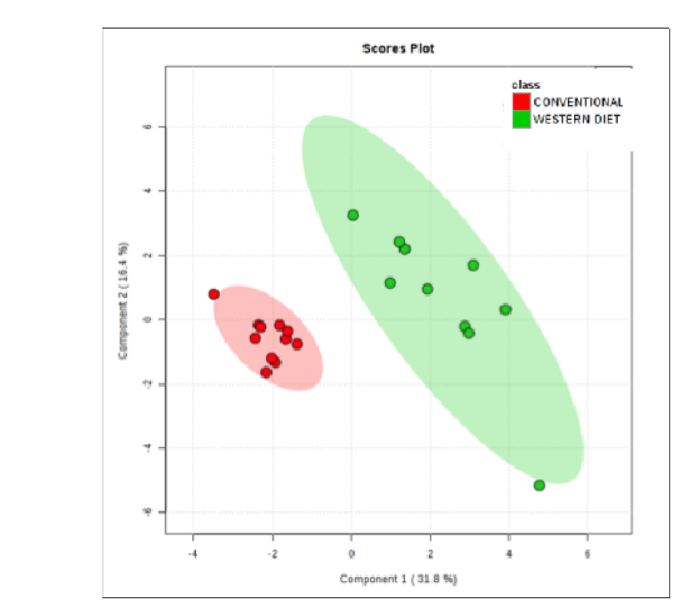

\section{B}

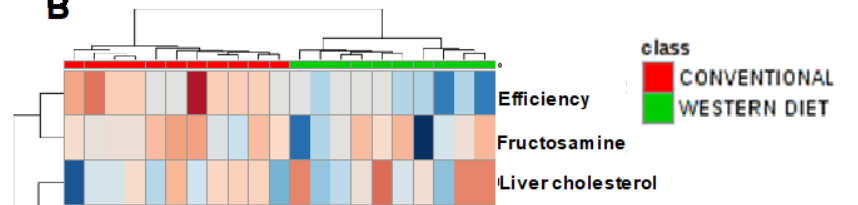

LDL Chol

Pelvicorenal fat

Total Chol

Growth rate

Final weight

Intermusc fat

Calorie intake

Total fat

Subcutaneous

\begin{tabular}{l|l|l|l|l|l|}
\hline Measure & 1 comps & 2 comps & 3 comps & 4 comps & 5 comps \\
\hline Accuracy & 0.95238 & 1.0 & 1.0 & 1.0 & 1.0 \\
\hline $\mathrm{R} 2$ & 0.82043 & 0.94066 & 0.96147 & 0.97133 & 0.98723 \\
\hline $\mathrm{Q} 2$ & 0.7679 & 0.87783 & 0.8926 & 0.89307 & 0.85764 \\
\hline
\end{tabular}

C

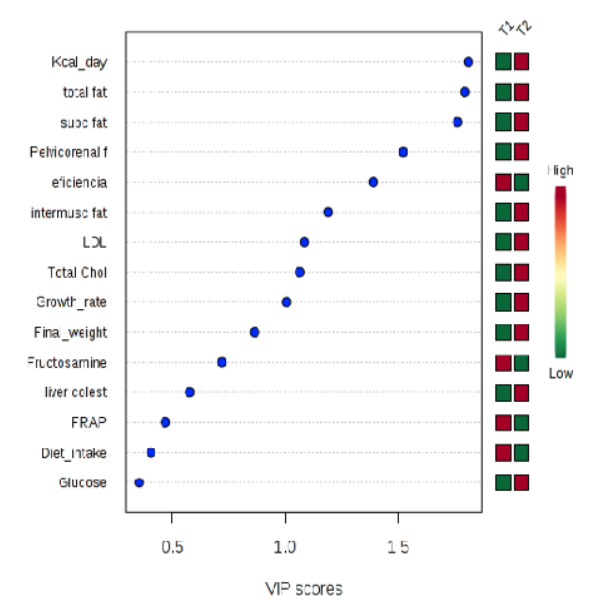

D

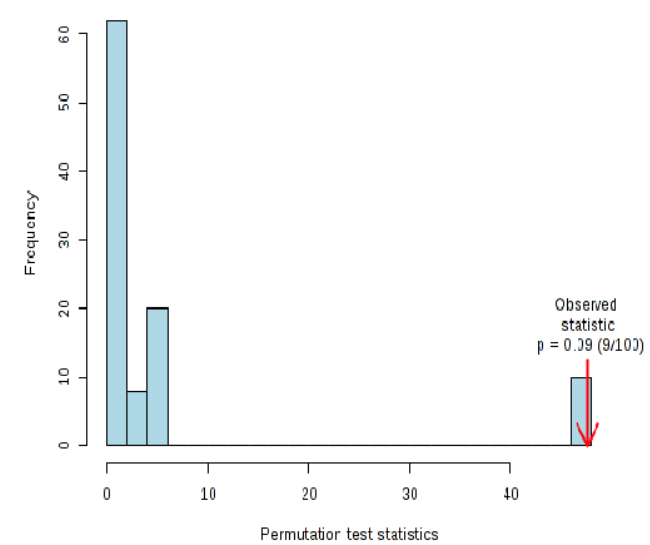


171 Supplemental Figure 2. Biochemical and morphological traits define an obesity signature in

172 the proposed model of prepubertal obesity. A) PLS-DA model demonstrating a high accuracy

173 of the model, with more than $30 \%$ of variance being explained by the first component. B)

174 Hierarchical clustering and heatmap shows that conventional (clinical biochemistry) and CT

175 measures define a signature induced by western-type diet and demonstrate fat depot

176 segregation according to anatomical location and clusterization with biochemical traits. C)

177 Variable importance in projection (VIP) Scores of biochemical and morphological traits in the

178 first component of the PLS-DA model. D) Permutation test of the model, showing a global $p$ 179 value with a 0.09 value.

\section{Supplemental Figure 3}

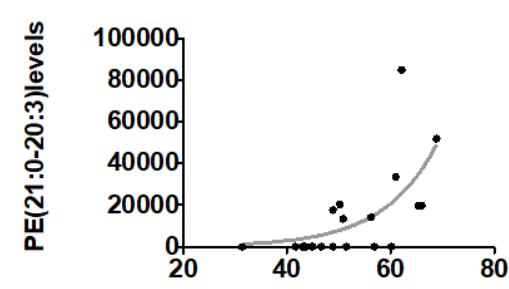

LDL-Cholesterol (mg/dL)

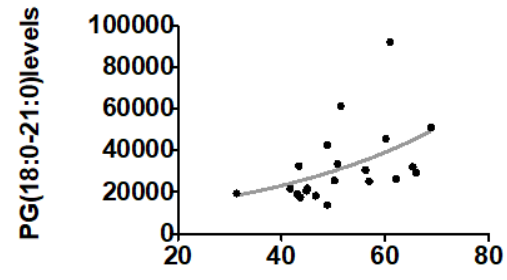

LDL-Cholesterol (mg/dL)

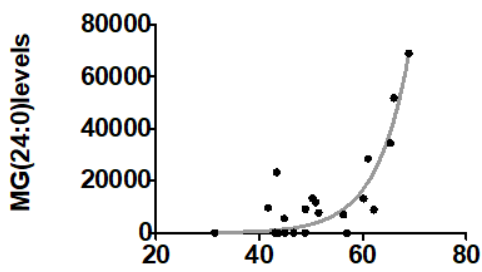

LDL-Cholesterol (mg/dL)

Supplemental Figure 3. Plasma LDL cholesterol values are associated with specific plasma

182 lipids. Association of plasma concentration of selected lipids with LDL-cholesterol concentration. Shown lipids are a selection from those showing a significant correlation with specific traits (at least $p<0.05$ by Spearman's rank correlation test, see Supplemental Data for

185 the whole list), while lines indicate non-linear fit (exponential growth equations; $186 Y=Y 0^{*} \exp \left(k^{*} X\right)$. 
A
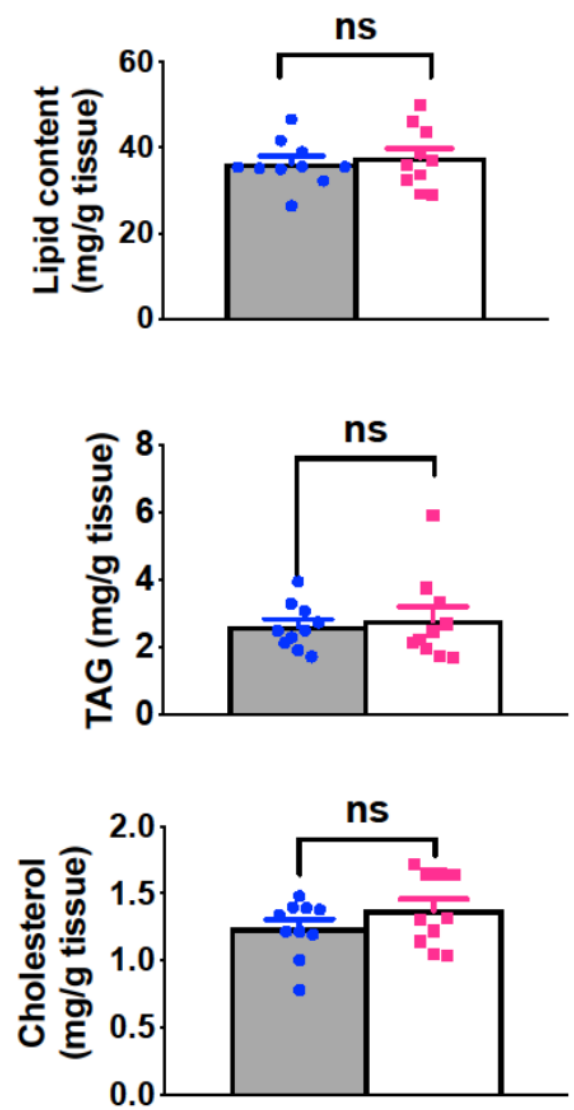

B

- Conventional

- Western diet

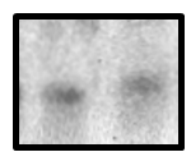

Blot Ab: pIRS1

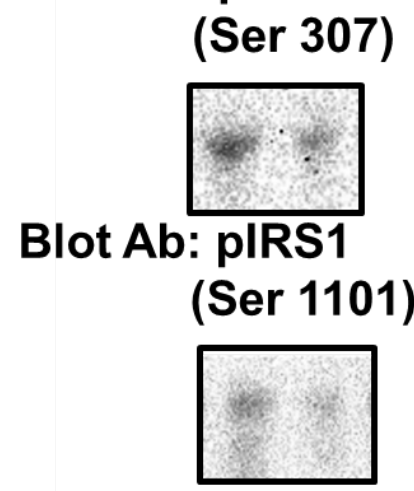

Blot Ab: pJun

(Ser 73)

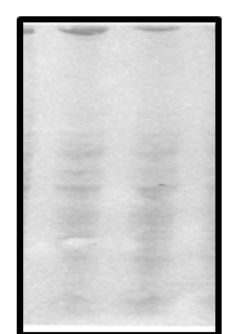

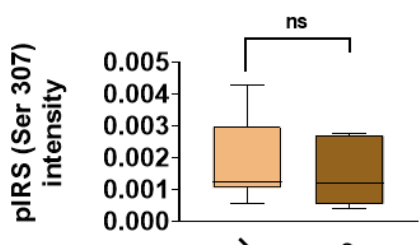

\section{Coomassie}

190 Supplemental Figure 4. A) Western-type diet does not increase lipid content in the liver,

191 nor triacylglyceride (TAG) concentrations or cholesterol levels B) shows a representative

192 western-blot of insulin-activity modulating signals in the liver and the influence of western-

193 type diet. Right graphs after each blot show densitometric integrated values normalized to

194 the total protein content, estimated by Coomassie blue staining, with $p$ values after Students

195 t-test ( $n=6$ for each group)

196 


\section{Skeletal muscle}

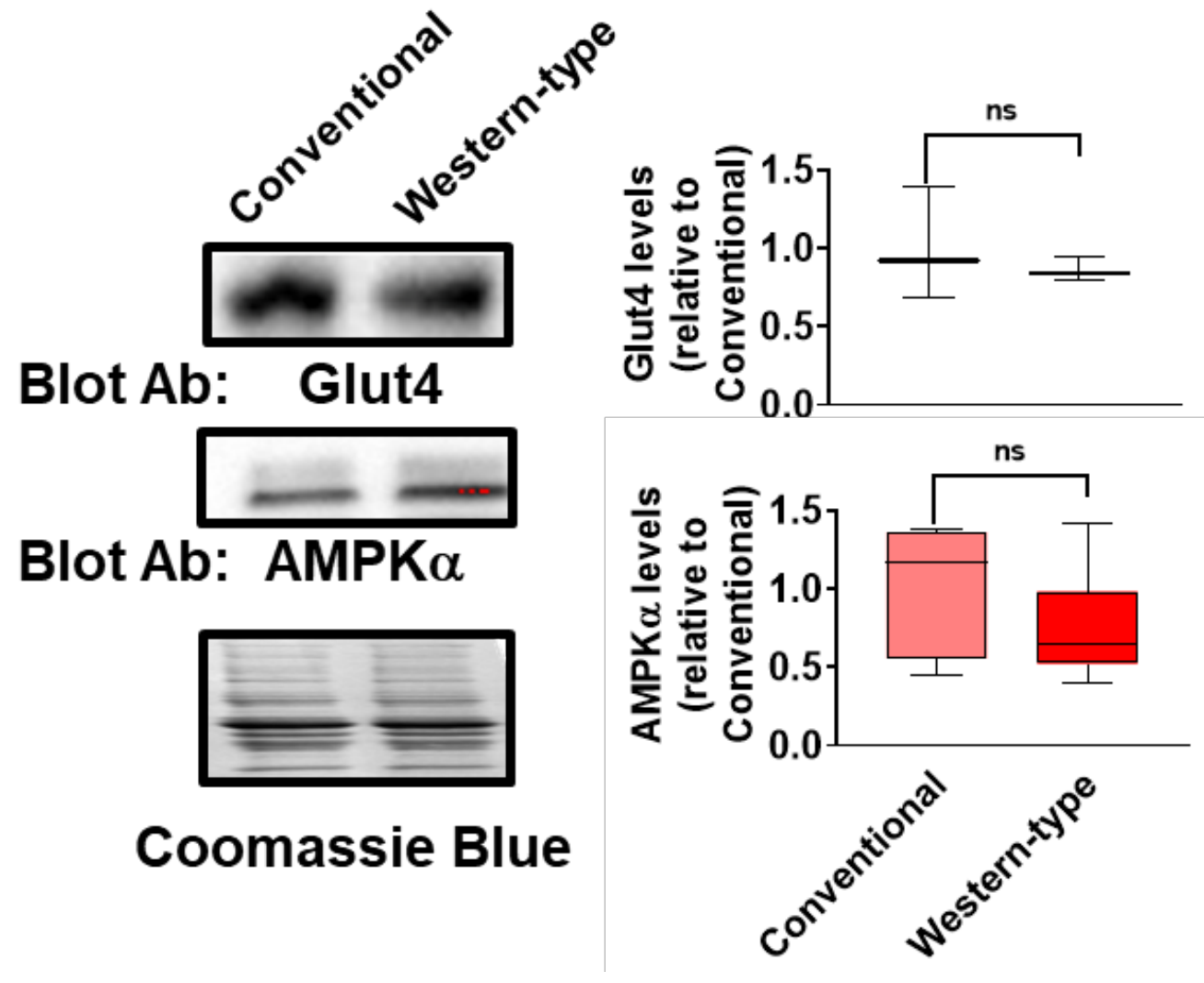

Supplemental Figure 5. Skeletal muscle insulin-responsive signals are not affected by

200 the western-type diet in the prepubertal model. Representative western-blot of insulin-

201 responsive signals in skeletal muscle. Right graphs show densitometric integrated values,

202 normalized to the intensity of animals fed with conventional diets all adjusted to total protein

203 content, estimated by Coomassie blue staining, with $p$ values after Students t-test 


\section{Supplemental Figure 6}

A
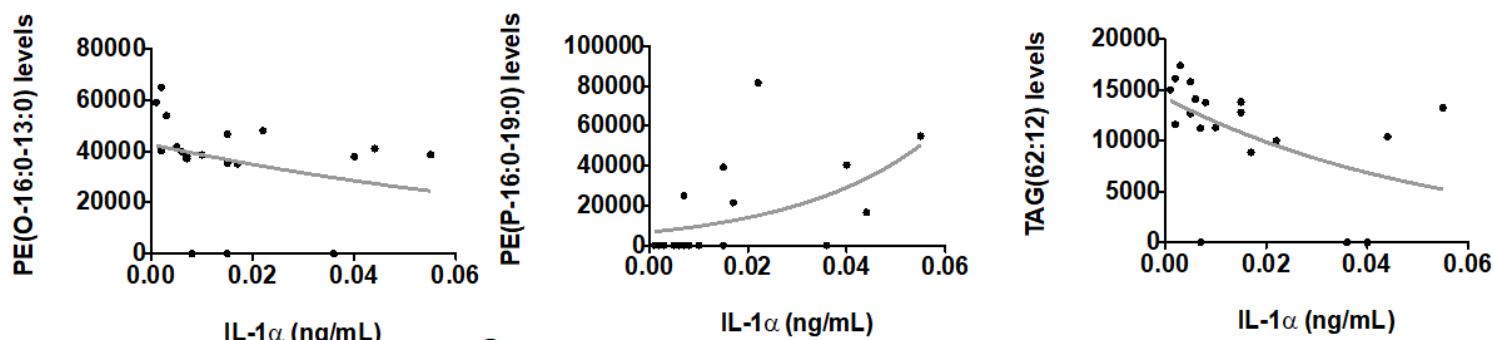

204

B

$\mathrm{IL}-1 \alpha(\mathrm{ng} / \mathrm{mL}) \quad$ C

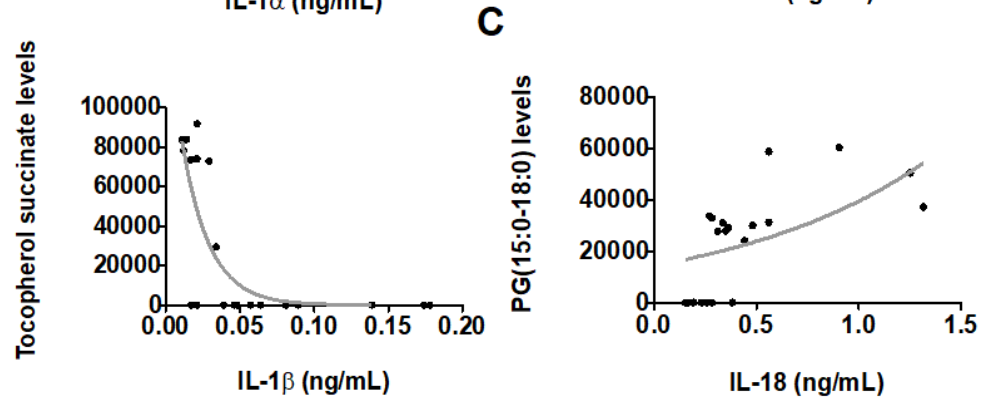

205 Supplemental Figure 6. Peripheral markers of inflammation are associated with specific

206 plasma lipids. Association of plasma concentration of selected lipids with individual IL1a (A),

207 IL1b (B) and IL-18 (C) Shown lipids are a selection from those showing significant correlation

208 with specific traits (at least $p<0.05$ by Spearman's rank correlation test, see Supplemental

209 Data for the whole list) while lines indicate non-linear fit (exponential growth equations;

$210 Y=Y 0^{*} \exp \left(k^{*} X\right)$. 


\section{Supplemental Figure 7}
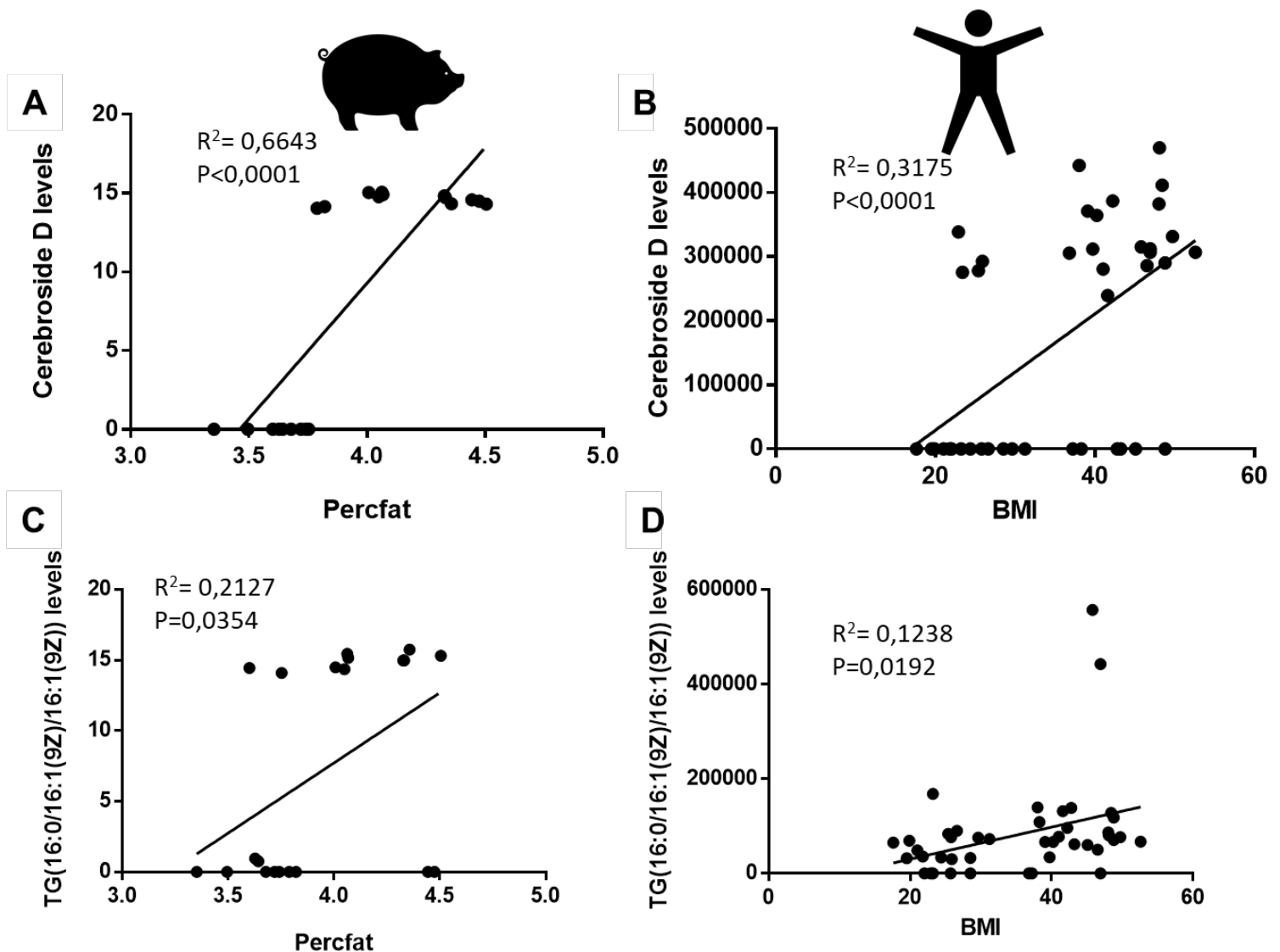

213 Supplemental Figure 7. Validation in humans with obesity of proposed biomarkers. Specific

214 lipids show a similar positive correlation with BMI (in humans, in B and D) and AT abundance

215 (in the porcine model, in $\mathrm{A}$ and $\mathrm{C}$ ).. All correlations are significant (at least $\mathrm{p}<0.05$ ) by

216 Spearman's rank correlation test. Lines show the linear relationship, with $R^{2}$ and $p$ values for

217 linear adjustment shown in the inset. Pig icon comes from aLf; and Human one by Ma Qing

218 both from the Noun Project (thenountproject.com) 


\section{Supplemental Figure 8}
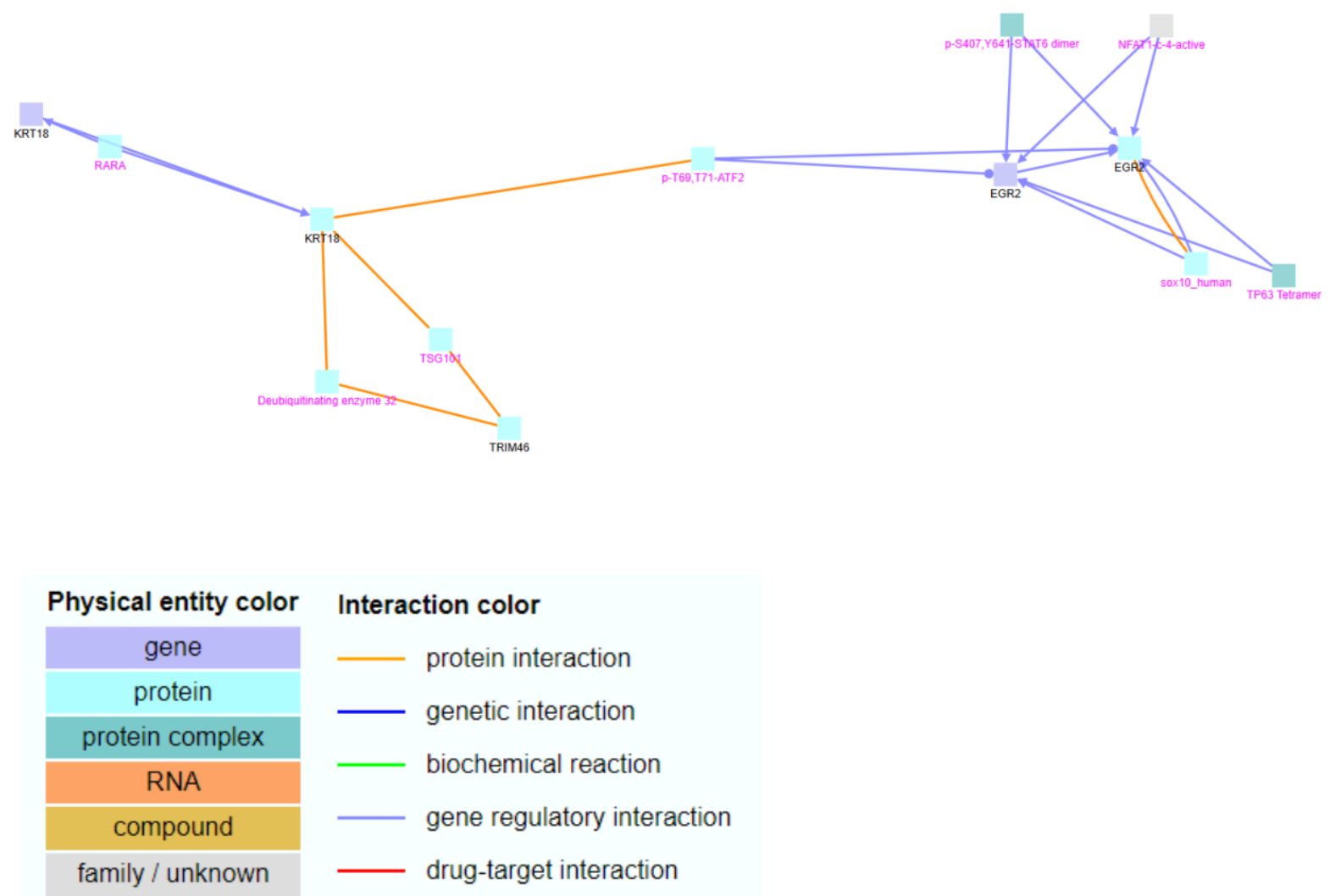

220 Supplemental Figure 8. Induced network module analyses of PBMC transcriptomic changes 221 associated with the western-type diet. Genes (see main text) were entered into the

222 ConsensusPathDB platform, and interactions, coded by colors link nodes, representing 223 entities (identified by colors). Black node labels denote genes significantly influenced by high224 calorie diet, while magenta node labels denote intermediate nodes. All types of protein 225 interactions (high to low confidence protein interactions) were considered, as well as genetic, 226 biochemical, and gene regulatory interactions, with all available database sources in the 227 platform being considered. 


\section{Supplemental Figure 9}

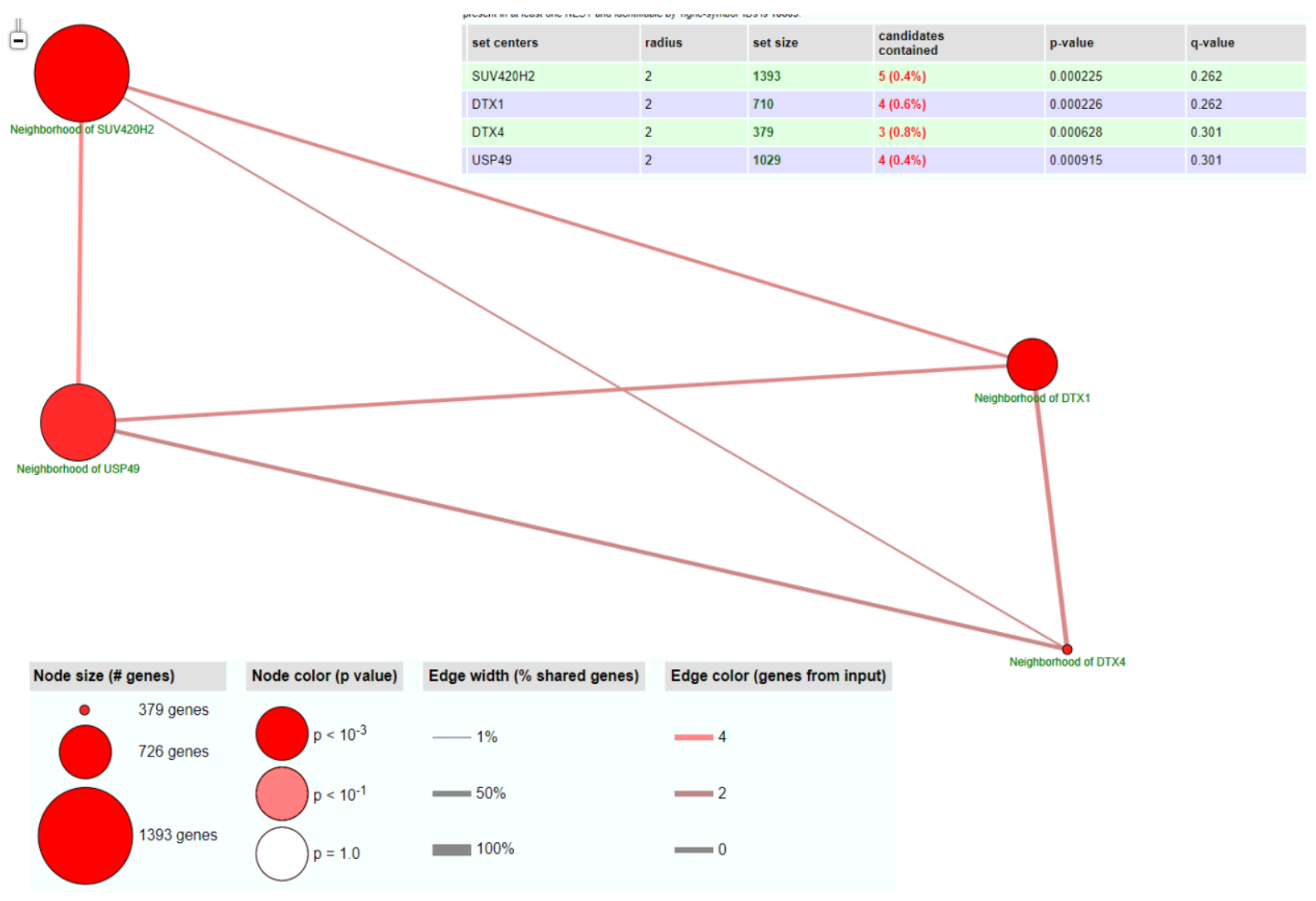

229 Supplemental Figure 9. Network neighborhood-based entity sets of PBMC transcriptomic 230 changes associated with western-type diet. Genes (see main text) were entered into

231 ConsensusPathDB platform, and nodes, representing neighborhood-based entity sets (whose

232 size is proportional to the number of genes of the set, and color intensity denotes $p$-value for

233 hypergeometric tests) are linked by interactions consisting of the number of genes shared by

234 nodes. The type of network chosen was 2-next neighbors, with a mínimum number of 2 genes

235 from PBMC transcriptome overlap with members of the entity set (and a $p<0.001$ as the cutoff).

236 The sets were obtained considering only Reactome based ones, with gene ontology level 5

237 categories and a p-value cutoff of 0.01 for ontology inclusión. The upper panel indicates the

238 statistical values of each entity set. 
Supplemental Figure 10

A

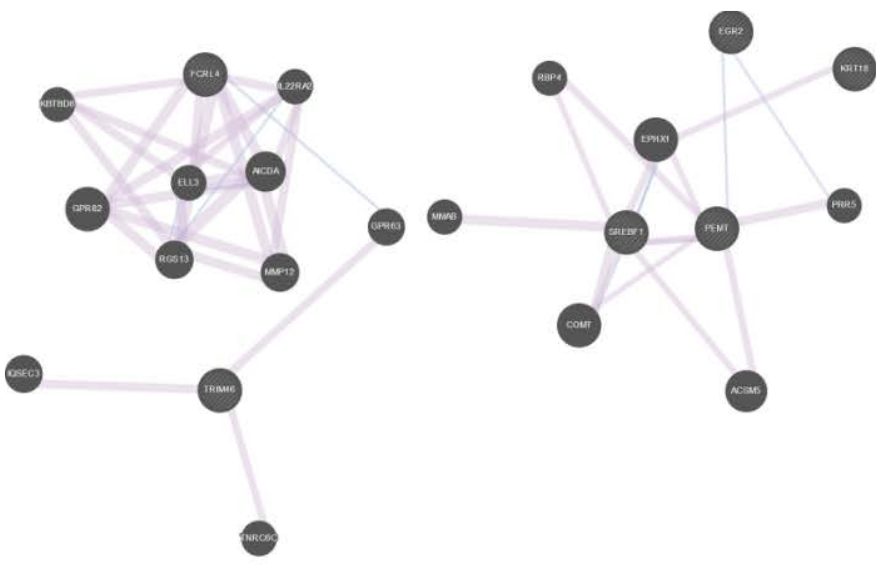

$+2$

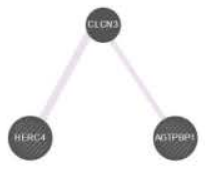

(20.

B

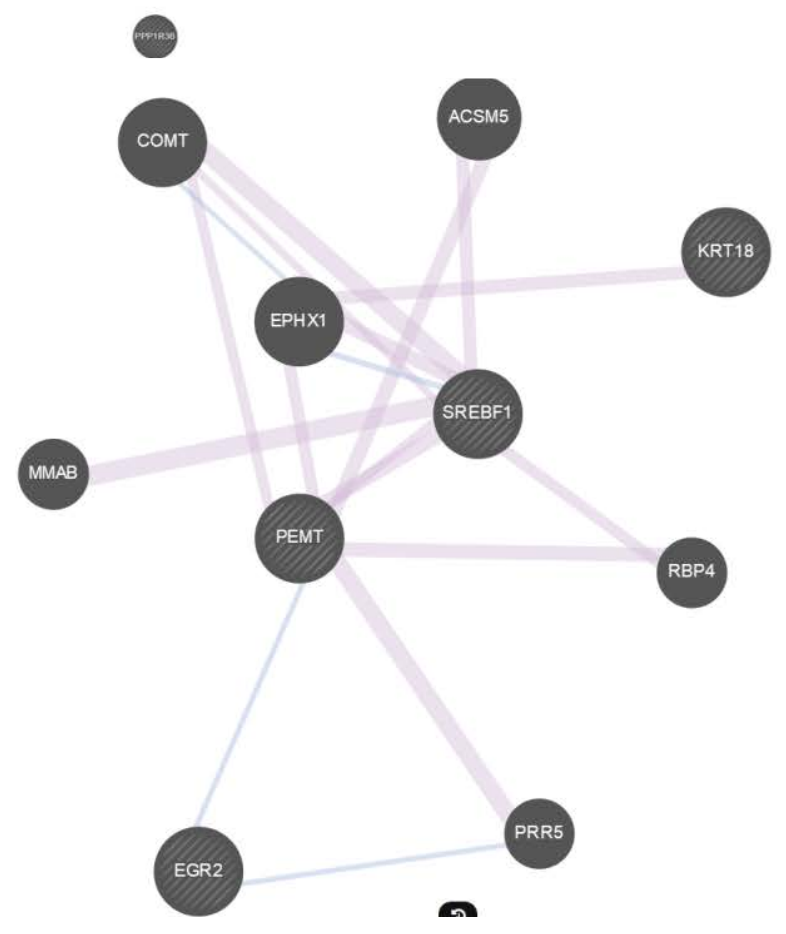


241 Supplemental Figure 10. Interatomic analyses of PBMC transcriptome changes.

242 Genes whose expression was changed by western-type diet in PBMC were introduced

243 into the Genemania platform, together with PEMT and SRBEP1, in order to evaluate

244 its potential clustering. The results (A) show five significant co-expression networks

245 and co-localization, with PEMT and SRBEP1 being localized into a network with EGR2

246 and KRT18.

247 
249 Supplemental Table 1. Clinical characteristics of the cohort used for translational 250 applicability.

\begin{tabular}{|l|l|}
\hline Parameter & Value $^{1}$ \\
\hline $\mathrm{N}$ & 44 \\
\hline Age & $49.41 \pm 8.71$ \\
\hline Gender & $52 \%$ female \\
\hline BMl $\left(\mathrm{Kg} / \mathrm{m}^{2}\right)$ & $35.04 \pm 10.7$ \\
\hline Waist to hip ratio & $0.90 \pm 0.11$ \\
\hline HOMA-IR & $3.08 \pm 3.07$ \\
\hline Blood Cholesterol (mg/dL) & $196.2 \pm 40.35$ \\
\hline Blood LDL-Cholesterol $(\mathrm{mg} / \mathrm{dL})$ & $122.9 \pm 34.6$ \\
\hline Blood HDL-Cholesterol $(\mathrm{mg} / \mathrm{dL})$ & $54.6 \pm 15.5$ \\
\hline Glycemia (mg/dL) & $94.2 \pm 12.5$ \\
\hline Triacylglyceridemia $(\mathrm{mg} / \mathrm{dL})$ & $95.5 \pm 49.49$ \\
\hline
\end{tabular}

${ }^{1}$ Values shown are mean \pm SD unless stated otherwise.

Supplemental Table 2. Composition of experimental diets applied from 9 to 11 weeks 254 of age

\begin{tabular}{|l|l|l|}
\hline & & \\
\hline Component(\%) & Conventional transition & Western-type transition \\
\hline Oats & 66.00 & - \\
\hline Wheat & - & 42.00 \\
\hline Maize & - & 16.00 \\
\hline Soy protein concentrate & - & - \\
\hline
\end{tabular}




\begin{tabular}{|c|c|c|}
\hline Hydrolyzed rice protein & - & - \\
\hline Caseinate & 10.96 & 9.88 \\
\hline Starch & 1.00 & 10.00 \\
\hline Saccharose & - & 5.00 \\
\hline Wheat bran & 15.00 & 4.00 \\
\hline Soybean hulls & 4.10 & - \\
\hline Lard & - & 10.00 \\
\hline Dicalcium phosphate & 1.18 & 1.52 \\
\hline Calcium carbonate & 0.98 & 0.51 \\
\hline Salt & 0.35 & 0.37 \\
\hline L-Lysine-HCl & - & 0.19 \\
\hline L-Threonine & - & 0.03 \\
\hline DL-Methionine & - & 0.10 \\
\hline L-Tryptophan & - & - \\
\hline Ethoxiquin & 0.02 & 0.02 \\
\hline Vit-Min complex $\left(^{*}\right)$ & 0.40 & 0.40 \\
\hline
\end{tabular}

$256\left(^{\star}\right)$ Provides per kg feed: vitamin A (E-672) 5500 Ul; vitamin D3 (E-671) 1100 Ul; vitamin E (alfa-tocopherol) 7 mg; vitamin B1 0.5

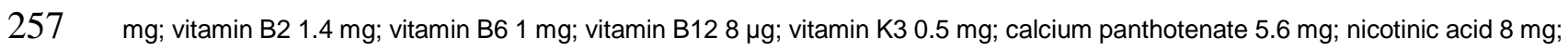
258 choline $120 \mathrm{mg}$; Fe (E-1) (from FeSO4.7H2O) 80 mg; I (E-2) (from Ca(I2O3)2) 0.5 mg; Co (E-3) (from 2CoCO3.3Co(OH)2.H2O) $2590.4 \mathrm{mg}$; $\mathrm{Cu}$ ( E-4) (from CuSO4.5H2O) $5 \mathrm{mg}$; Cu (E-4) (from amino acids quelate) $5 \mathrm{mg}$; Mn (E-5) (from MnO) $40 \mathrm{mg} ; \mathrm{Zn}$ (E-6) 260 (from $\mathrm{ZnO}) 100 \mathrm{mg}$; Se (E-8) (from Na2SeO3) $0.25 \mathrm{mg}$.

261 Supplemental Table 3. Nutritional composition of experimental diets applied from 9

262 to 11 weeks of age

\begin{tabular}{|l|l|l|}
\hline Amount (\%) & Conventional transition & Western-type transition \\
\hline Crude Protein & 17.98 & 15.55 \\
\hline Crude Fibre & 12.20 & 1.68 \\
\hline Fat & 4.98 & 11.80 \\
\hline Ash & 5.96 & 4.15 \\
\hline
\end{tabular}




\begin{tabular}{|l|l|l|}
\hline Sodium & 0.15 & 0.15 \\
\hline Chloride & 0.29 & 0.31 \\
\hline Total calcium & 0.86 & 0.70 \\
\hline Total phosphorous & 0.58 & 0.49 \\
\hline Digestible phosphorous & 0.27 & 0.27 \\
\hline Lysine & 1.11 & 1.03 \\
\hline Threonine & 0.75 & 0.65 \\
\hline Methionine & 0.41 & 0.44 \\
\hline Met+Cys & 0.69 & 0.64 \\
\hline Tryptophan & 0.26 & 0.22 \\
\hline Energy (MJ ME/kg) & 10.42 & 15.39 \\
\hline & & \\
\hline
\end{tabular}

264 Supplemental Table 4. Composition of experimental diets applied from 12 to 19

265 weeks of age

\begin{tabular}{|l|l|l|}
\hline Component(\%) & Conventional & Western-type \\
\hline Oats & 55.28 & - \\
\hline Wheat & - & 45.87 \\
\hline Maize & - & 13.56 \\
\hline Caseinate & 9.76 & 8.54 \\
\hline Starch & 13.13 & 10.00 \\
\hline Saccharose & - & 5.00 \\
\hline Wheat bran & 15.00 & 4.00 \\
\hline Soybean hulls & 4.00 & - \\
\hline Lard & - & 10.00 \\
\hline Dicalcium phosphate & 1.27 & 1.51 \\
\hline
\end{tabular}




\begin{tabular}{|l|l|l|}
\hline Calcium carbonate & 0.78 & 0.51 \\
\hline Salt & 0.36 & 0.37 \\
\hline Ethoxiquin & 0.02 & 0.02 \\
\hline L-Lysine-HCL & - & 0.15 \\
\hline L-Threonine & - & 0.01 \\
\hline DL-Methinonine & 0.003 & 0.07 \\
\hline Vit-Min complex $\left(^{*}\right)$ & 0.40 & 0.40 \\
\hline
\end{tabular}

$266\left(^{*}\right)$ Provides per kg feed: vitamin A (E-672) 5500 UI; vitamin D3 (E-671) 1100 UI; vitamin E (alfa-tocopherol) 7 mg; vitamin B1 0.5 mg;

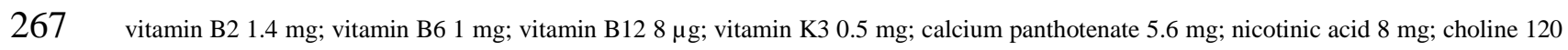

268 mg; Fe (E-1) (from FeSO4·7H2O) 80 mg; I (E-2) (from Ca(I2O3)2) 0.5 mg; Co (E-3) (from 2CoCO3·3Co(OH)2·H2O) 0.4 mg; Cu ( E-4)

269 (from CuSO4·5H2O) 5 mg; Cu (E-4) (from amino acids quelate) 5 mg; Mn (E-5) (from MnO) 40 mg; Zn (E-6) (from ZnO) 100 mg; Se (E-8) (from Na2SeO3) $0.25 \mathrm{mg}$. 
273 Supplemental Table 5. Nutritional composition of experimental diets applied from 12

274 to 19 weeks of age

\begin{tabular}{|l|l|l|}
\hline Amount(\%) & Conventional & Western-type \\
\hline Protein & 17.6 & 14.34 \\
\hline Fat & 4.6 & 11.55 \\
\hline Fiber & 11 & 1.72 \\
\hline Sodium & 0.15 & 0.15 \\
\hline Chloride & 0.29 & 0.31 \\
\hline Calcium & 0.80 & 0.70 \\
\hline Total phosphorous & 0.56 & 0.49 \\
\hline Digestible phosphorous & 0.27 & 0.27 \\
\hline Lysine & 0.99 & 0.90 \\
\hline Threonine & 0.66 & 0.57 \\
\hline Methionine & 0.36 & 0.37 \\
\hline Met+Cys & 0.61 & 0.56 \\
\hline Tryptophan & 0.23 & 0.20 \\
\hline Ash & 5.55 & 4.13 \\
\hline Energy (MJ/kg) & 10.83 & 15.16 \\
\hline
\end{tabular}

275

276 
279 Supplemental Table 6. Antibodies and conditions.

280

\begin{tabular}{|l|l|l|l|}
\hline Antigen & Supplier & Reference & Dilution \\
\hline GLUT2 & Abcam & ab104622 & $1: 1000$ \\
\hline SREBP1 & Abcam & ab3259 & $1: 1000$ \\
\hline GLUT4 & Invitrogen & MA1-83191 & $1: 1000$ \\
\hline AMPK $\alpha$ & Invitrogen & PA5-17398 & $1: 1000$ \\
\hline Phospho-IRS1 (Ser & Upstate & $07-247$ & $1: 1000$ \\
\hline 307$)$ & & & \\
\hline Phospho-IRS1 (Ser & Cell Signaling & 2385 & $1: 300$ \\
\hline 1101) & & & $1: 1000$ \\
\hline Phospho-c-JUN & Cell Signaling & 3270 & \\
\hline (Ser73) & & & $1: 100000$ \\
\hline anti-mouse IgG & Amersham & NA931V & \\
\hline anti-rabbit IgG & Pierce & 31460 & \\
\hline
\end{tabular}


Supplemental Table 7. Internal standards for lipidomic analyses.

\begin{tabular}{|c|c|c|c|}
\hline Compound & Reference & & \\
\hline 1,3(d5)-dihexadecanoyl-glycerol & $\begin{array}{l}\text { 110537, } \\
\text { Lipids }\end{array}$ & Avanti & Polar \\
\hline 1,3(d5)-dihexadecanoyl-2-octadecanoyl-glycerol & $\begin{array}{l}\text { 110543, } \\
\text { Lipids }\end{array}$ & Avanti & Polar \\
\hline $\begin{array}{l}\text { 1-hexadecanoyl(d31)-2-(9Z-octadecenoyl)-sn- } \\
\text { glycero-3-phosphate }\end{array}$ & $\begin{array}{l}\text { 110920, } \\
\text { Lipids }\end{array}$ & Avanti & Polar \\
\hline $\begin{array}{l}\text { 1-hexadecanoyl(d31)-2-(9Z-octadecenoyl)-sn- } \\
\text { glycero-3-phosphocholine }\end{array}$ & $\begin{array}{l}\text { 110918, } \\
\text { Lipids }\end{array}$ & Avanti & Polar \\
\hline $\begin{array}{l}\text { 1-hexadecanoyl(d31)-2-(9Z-octadecenoyl)-sn- } \\
\text { glycero-3-phosphoethanolamine }\end{array}$ & $\begin{array}{l}\text { 110921, } \\
\text { Lipids }\end{array}$ & Avanti & Polar \\
\hline $\begin{array}{l}\text { 1-hexadecanoyl-2-(9Z-octadecenoyl)-sn-glycero-3- } \\
\left.\text { phospho-(1'-rac-glycerol-1', } 1^{\prime}, 2^{\prime}, 3^{\prime}, 3^{\prime}-d 5\right)\end{array}$ & $\begin{array}{l}\text { 110899, } \\
\text { Lipids }\end{array}$ & Avanti & Polar \\
\hline $\begin{array}{l}\text { 1-hexadecanoyl(d31)-2-(9Z-octadecenoyl)-sn- } \\
\text { glycero-3-phospho-myo-inositol }\end{array}$ & $\begin{array}{l}\text { 110923, } \\
\text { Lipids }\end{array}$ & Avanti & Polar \\
\hline $\begin{array}{l}\text { 1-hexadecanoyl(d31)-2-(9Z-octadecenoyl)-sn- } \\
\text { glycero-3-[phospho-L-serine] }\end{array}$ & $\begin{array}{l}\text { 110922, } \\
\text { Lipids }\end{array}$ & Avanti & Polar \\
\hline 26:0-d4 Lyso PC & $\begin{array}{l}860389, \\
\text { Lipids }\end{array}$ & Avanti & Polar \\
\hline 18:1 Cholesterol $(d 7)$ ester & $\begin{array}{l}\text { 111015, } \\
\text { Lipids }\end{array}$ & Avanti & Polar \\
\hline cholest-5-en-3ß-ol( $(d 7)$ & $\begin{array}{l}\text { LM-4100, } \\
\text { Lipids }\end{array}$ & Avanti & Polar \\
\hline
\end{tabular}




\begin{tabular}{|llll|}
\hline D-erythro-sphingosine- $d 7$ & 860657, & Avanti & Polar \\
& Lipids & & \\
D-erythro-sphingosine- $d 7$-1-phosphate & 860659, & Avanti & Polar \\
& Lipids & & \\
N-palmitoyl-d31-D-erythro-sphingosine & 868516, & Avanti & Polar \\
& Lipids & & \\
N-palmitoyl-d31-D-erythro- & 868584, & Avanti & Polar \\
sphingosylphosphorylcholine & Lipids & & \\
Octadecanoic acid-2,2- $d 2$ & $19905-58-9$, Sigma Aldrich \\
\end{tabular}

Supplemental Table 8 . Changes in subcutaneous AT mRNA concentration induced by the western-type diet.

\begin{tabular}{|l|l|l|l|l|}
\hline Gene & Conventional $^{1}$ & Western-type & Change (\%) & $p$-value \\
\hline lep & $0.0225 \pm 0.0152$ & $0.064 \pm 0.0215$ & 184.8 & $<0.00001$ \\
\hline II6 & $0.0072 \pm 0.0079$ & $0.0032 \pm 0.0015$ & -55.7 & 0.15 \\
\hline Irs1 & $0.0635 \pm 0.0291$ & $0.0464 \pm 0.0199$ & -26.9 & 0.158 \\
\hline fasn & $5.4763 \pm 1.732$ & $5.5608 \pm 1.4093$ & 1.5 & 0.908 \\
\hline adipoq & $126.87 \pm 71.05$ & $94.71 \pm 34.74$ & -25.3 & 0.211 \\
\hline Insr1 & $1.05 \pm 0.22$ & $1.13 \pm 0.22$ & 7.6 & 0.455 \\
\hline gapdh & $17.77 \pm 4.53$ & $20.9 \pm 7.29$ & 17.6 & 0.247 \\
\hline
\end{tabular}

${ }^{1}$ Values shown are mean \pm SD unless stated otherwise. 
294 Supplemental Table 9. Changes in plasma interleukine concentration induced by the

295 western-type diet.

\begin{tabular}{|l|l|l|l|}
\hline $\begin{array}{l}\text { Parameter } \\
(\mathrm{pg} / \mathrm{mL})\end{array}$ & Conventional & Western-type & $\mathrm{p}^{1}$ \\
\hline $\mathrm{IL}-1 \mathrm{a}$ & $0.016 .9 \pm 0.014$ & $0.014 \pm 0.019$ & 0.9 \\
\hline IL-1b & $0.054 \pm 0.047$ & $0.056 \pm 0.061$ & 0.9 \\
\hline IL-1ra & $0.13 \pm 0.12$ & $0.11 \pm 0.11$ & 0.6 \\
\hline IL-10 & $0.08 \pm 0.12$ & $0.09 \pm 0.16$ & 0.9 \\
\hline IL-18 & $0.43 \pm 0.31$ & $0.48 \pm 0.36$ & 0.9 \\
\hline
\end{tabular}

296

297 Values shown are mean \pm SD unless stated otherwise. ${ }^{1}$ Student's t-test comparison

298 between values in conventional and western-type diets 
300 Supplemental Table 10. Changes in plasma metabolome induced by western-type diet

\begin{tabular}{|l|l|l|l|l|l|}
\hline Compound & $\begin{array}{l}\text { Log } \\
\text { FC }\end{array}$ & $p$ & $\begin{array}{l}\text { p } \\
\text { (Corr) }\end{array}$ & $\begin{array}{l}\text { Regulation } \\
\text { (Conventiona } \\
\text { l versus } \\
\text { western-type) }\end{array}$ & $\begin{array}{l}\text { FC } \\
\text { (Control } \\
\text { vs High } \\
\text { Calorie) }\end{array}$ \\
\hline Leucine & -0.44 & $\begin{array}{l}8.22 \mathrm{E} \\
-04\end{array}$ & $\begin{array}{l}2.73 \mathrm{E} \\
-02\end{array}$ & Up & 1.36 \\
\hline Pantheine & -1.35 & $\begin{array}{l}1.51 \mathrm{E} \\
-04\end{array}$ & $\begin{array}{l}8.38 \mathrm{E} \\
-03\end{array}$ & Up & 2.55 \\
\hline N-ethylarachidonoyl amine & - & $\begin{array}{l}2.16 \mathrm{E} \\
-04\end{array}$ & $\begin{array}{l}1.09 \mathrm{E} \\
-02\end{array}$ & Up & 27116.8 \\
& 14.7 & -04 & & 1 \\
\hline N-Lignoceroylsphingosine & -0.94 & $\begin{array}{l}9.37 \mathrm{E} \\
-04\end{array}$ & $\begin{array}{l}3.01 \mathrm{E} \\
-02\end{array}$ & Up & 1.92 \\
\hline $\begin{array}{l}\text { 3alpha,6alpha,7alpha,12alpha } \\
\text {-Tetrahydroxy-5beta-cholest- } \\
\text { 24-en-26-oic acid }\end{array}$ & 13.9 & $\begin{array}{l}1.30 \mathrm{E} \\
-05\end{array}$ & $\begin{array}{l}9.63 \mathrm{E} \\
-04\end{array}$ & Down & - \\
\hline GInMetGIn & 14.4 & $\begin{array}{l}4.82 \mathrm{E} \\
-08\end{array}$ & $\begin{array}{l}6.85 \mathrm{E} \\
-06\end{array}$ & Down & $\begin{array}{l}15602.2 \\
2\end{array}$ \\
\hline
\end{tabular}


Figure 2 whole western blots

Glut4 Omental Glut4 Subcut

Glut2 PelvRen
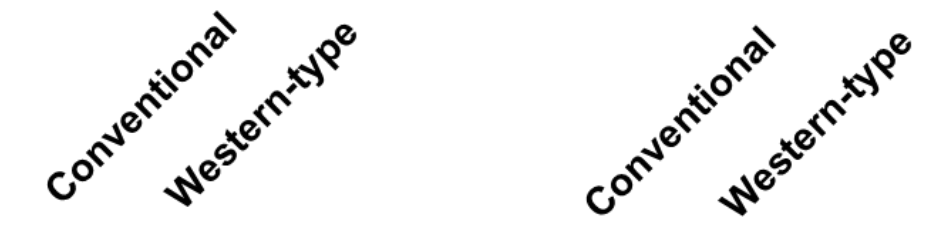

Srbep1 Subcut Srbep1 Oment
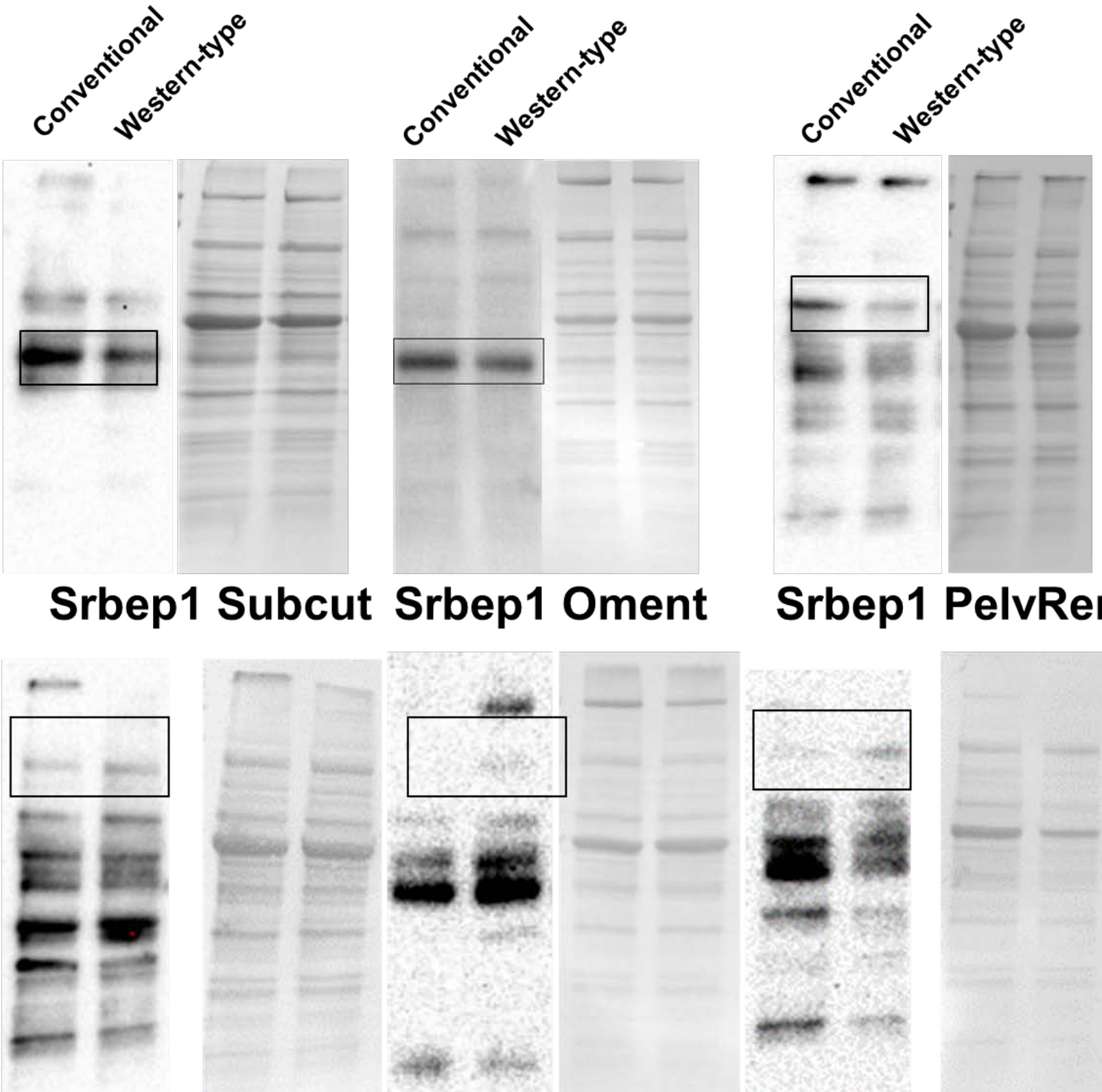

Srbep1 PelvRen
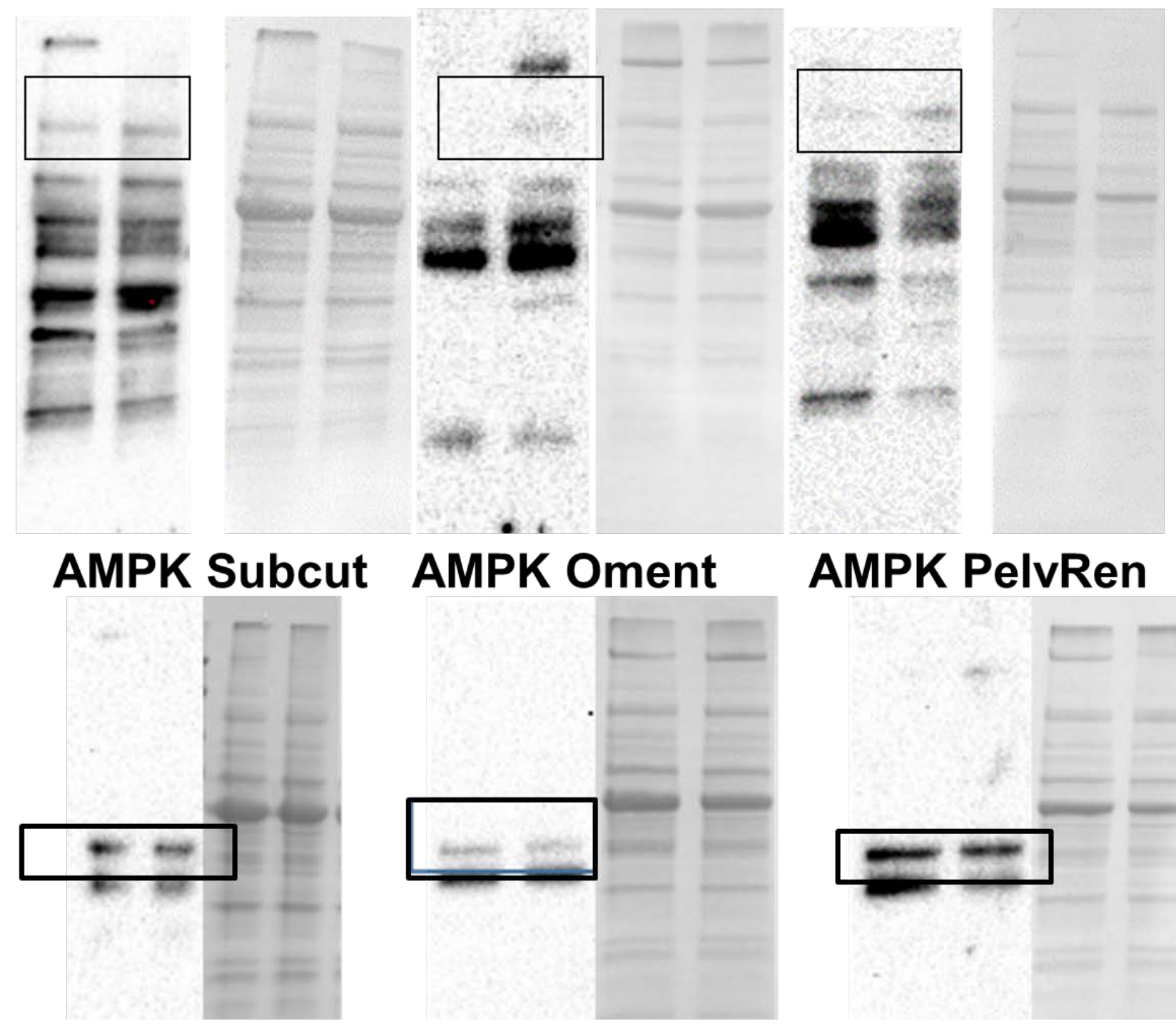
Figure 4 whole western blots

Glut4

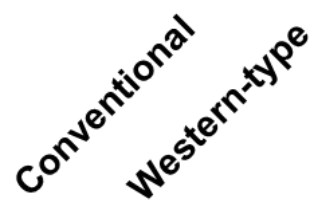

AMPK
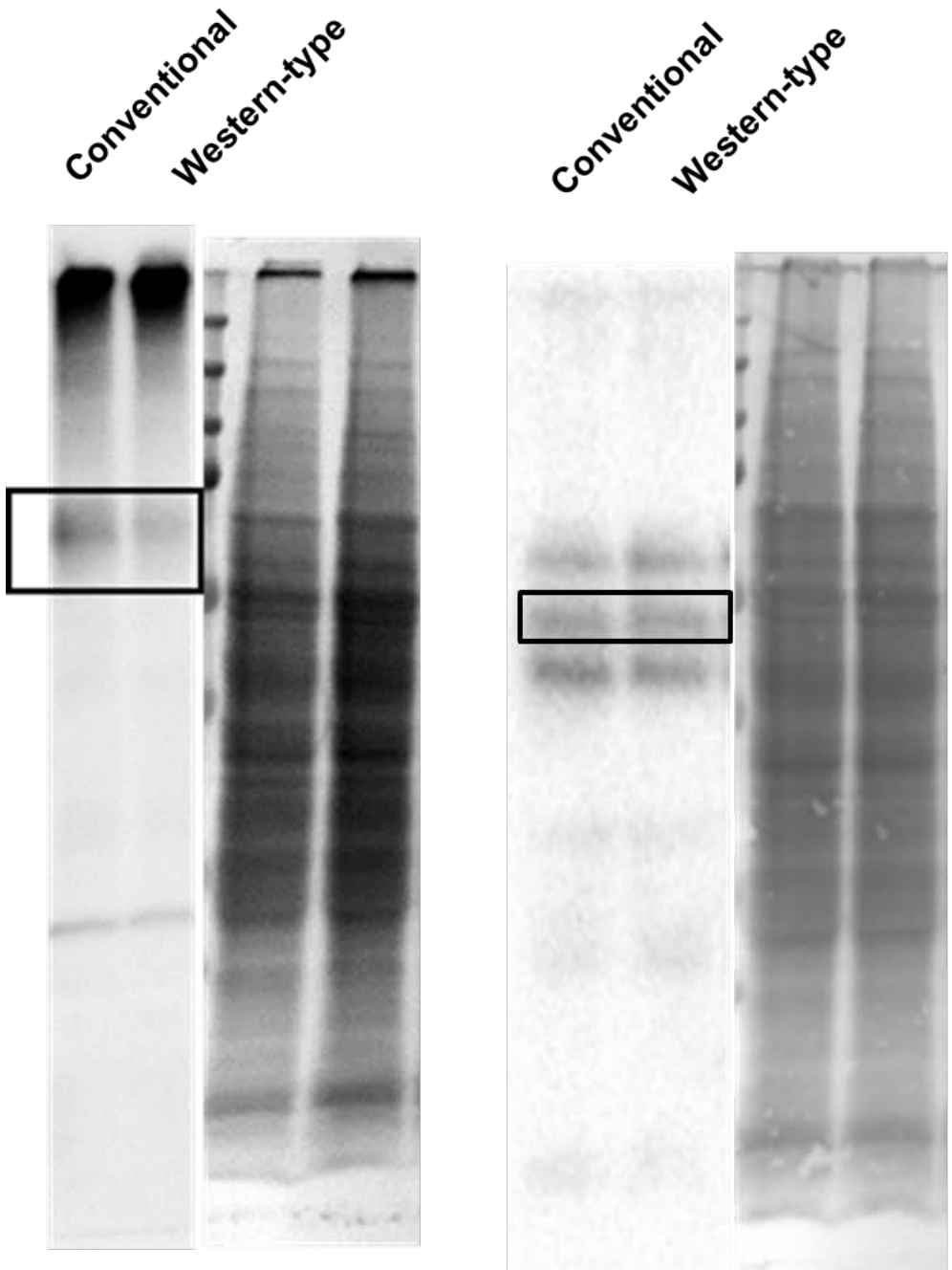

Srepb1
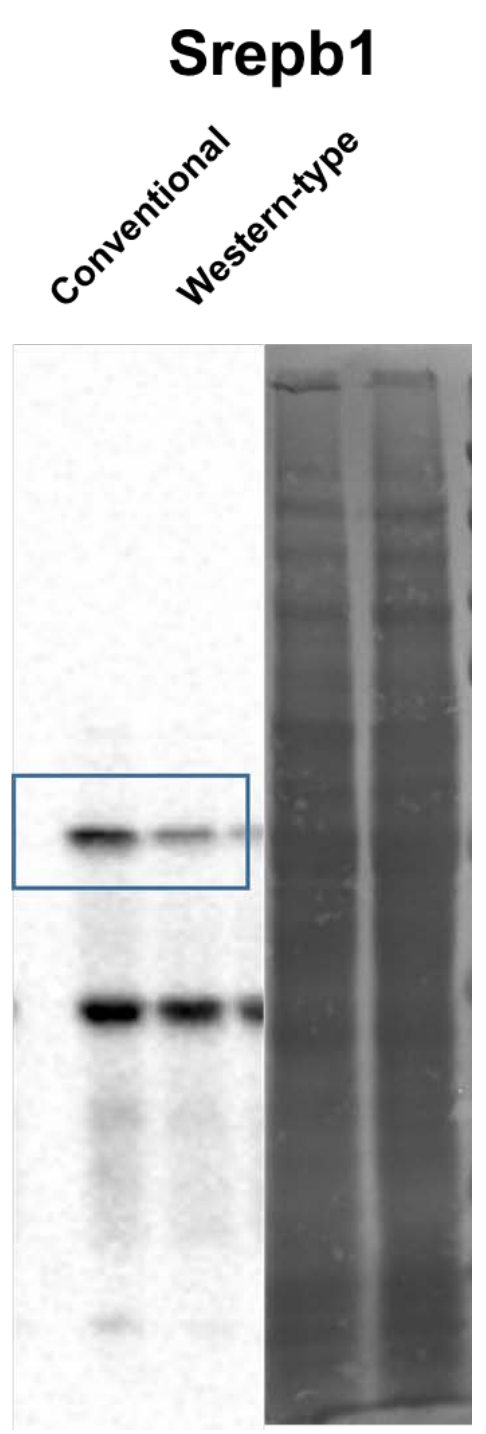
Supplemental Figure 4 whole western blots

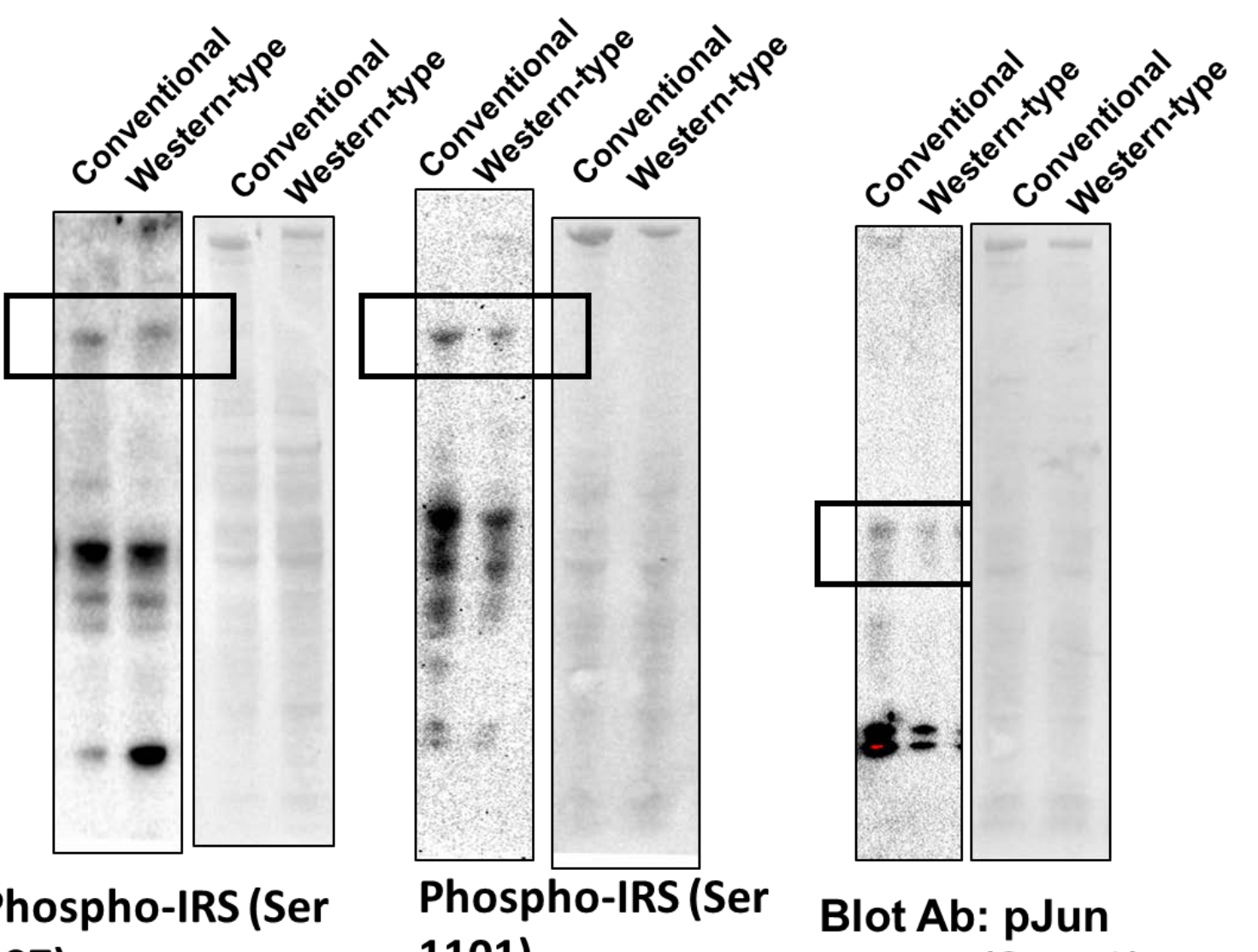

307)

Upstate 07-247
1101)

Upstate 07-247
(Ser 73) 
Supplemental Figure 5 whole western blots

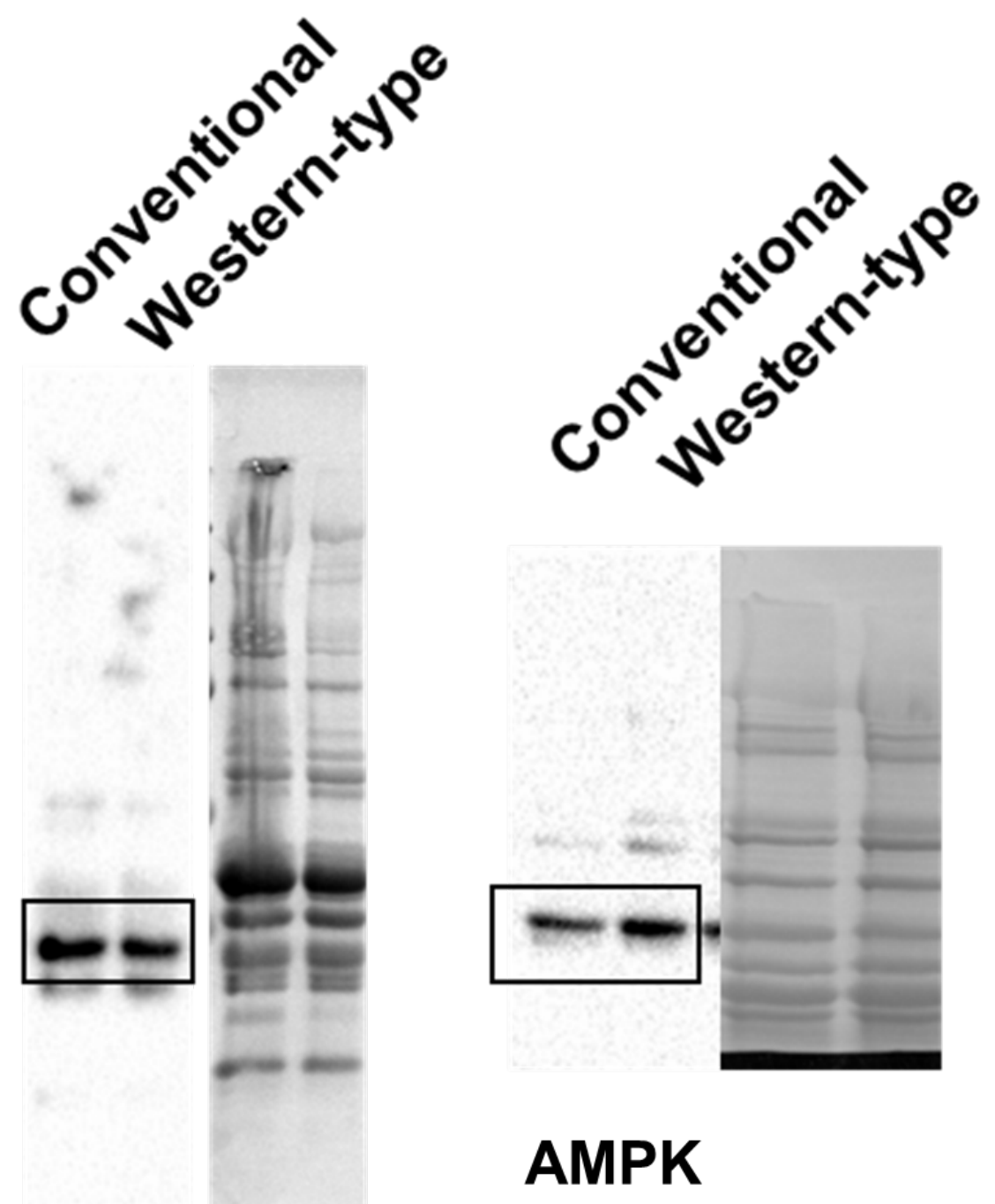

Glut4 